\title{
TOPOLOGICALLY, QUASICONFORMALLY OR LIPSCHITZ LOCALLY FLAT EMBEDDINGS IN CODIMENSION ONE
}

\author{
JOUNI LUUKKAINEN
}

\section{Introduction}

We consider locally $C$-flat embeddings in codimension one in three categories $C$ : TOP (continuous maps), LQC (locally quasiconformal maps), and LIP (locally Lipschitz maps). The latter two categories are our main subjects; the first one is in fact only auxiliary. In $\left[\mathrm{TV}_{1}\right],\left[\mathrm{V}_{3}\right],[\mathrm{LT}]$ the category $\mathrm{LQC}$ is extended to a category LQS of locally quasisymmetric maps: LQS manifolds and their LQS homeomorphisms are the same thing as LQC manifolds and their LQC homeomorphisms, but LQS embeddings are defined in every codimension. We consider LQS or LIP embeddings in codimension one (which need not be locally TOP flat), too. We refer to Section 2 for terminology. The prefix TOP will usually be omitted.

Sullivan [S] proved recently for $n \neq 4$ the existence and Hauptvermutung of $C$-structures, $C \in\{\mathrm{LQC}, \mathrm{LIP}\}$, on every $n$-manifold without boundary. Tukia and Väisälä $\left[\mathrm{TV}_{3}\right]$ then extended for $n \neq 4,5$ these results to all $n$-manifolds which may have a boundary. In the LQC case they made use of the extension of a quasiconformal selfhomeomorphism of $R^{n}$ to one of $R_{+}^{n+1}$, which they proved in [TV $\mathrm{TV}_{2}$ by the aid of $[\mathrm{S}]$.

In Section 3 we apply the basic result of $\left[\mathrm{TV}_{3}\right]$ on approximation of homeomorphisms by $C$-homeomorphisms, $C \in\{\mathrm{LQC}, \mathrm{LIP}\}$, to generalize it so as to give it the following form: The given homeomorphism $f$ is supposed to carry,

either homeomorphically or $C$-homeomorphically, a given closed locally $C$-flat submanifold $M$ of codimension one onto a submanifold of the same kind, and the approximating $C$-homeomorphism is claimed either to carry $M$ onto $f M$ or to coincide with $f$ on $M$, respectively.

In Section 4 we prove, in a relative form, for all $C \in\{$ TOP, LQC, LIP $\}$ and $n \neq 4$ that if two locally $C$-flat embeddings of an $(n-1)$-dimensional $C$-manifold into an $n$-dimensional $C$-manifold without boundary are sufficiently close to a given closed embedding, then one of them is carried onto the other by a small ambient isotopy consisting of $C$-homeomorphisms. For $C=$ TOP this absolute form is due to Craggs [Cr] if $n=3$ and to Price and Seebeck [PS] if $n \geqq 5$. We prove the 
relative form by applying $[\mathrm{Cr}]$ and $[\mathrm{PS}]$. For $C \neq \mathrm{TOP}$ we use the TOP result and Theorem 3.1.

Section 5 contains our main theorem: If $C \in\{$ TOP, LQC, LIP $\}$ and $n \neq 4$, every embedding of an $(n-1)$-dimensional $C$-manifold into an $n$-dimensional $C$-manifold without boundary can be relatively approximated by locally $C$-flat embeddings. The absolute case for $C=\mathrm{TOP}, n \geqq 5$ is a recent deep result of Ancel and Cannon $[\mathrm{AC}]$. The relative case follows from this result and Theorem 4.1. For $C=$ TOP, $n=3$ the theorem is due to Bing. For $C \neq$ TOP we apply the TOP case and results of Section 3. Our theorem has also an analogue for immersions.

In Section 6 we prove that if either $Y \subset X \subset R^{n-1}$ with $Y$ open in $R^{n-1}$ or $Y \subset X \subset R_{+}^{n-1}$ with $Y$ open in $R_{+}^{n-1}$ and if $n \neq 4$, then every embedding $f: X \rightarrow R^{n}$ which is quasisymmetric or bilipschitz, respectively, can be approximated on $Y$ by locally LIP flat embeddings which can be extended by $f \mid X \backslash Y$ to quasisymmetric embeddings or bilipschitz embeddings, respectively, of $X$. The proof is based on Theorem 5.3 on extending locally LIP flat approximations. An analogue of this result is proved in [LT]. There the dimension pair $(n-1, n), n \neq 4$, is replaced by a pair $(n, q)$ such that either $q \geqq n=1$ or $2 \leqq n \leqq q \leqq 3$ or $n \geqq 2, q \geqq n+3$ and PL (piecewise linear) embeddings are used in place of locally LIP flat embeddings.

In Section 7 we prove, using theorems of the two previous sections, that if $C \in\{\mathrm{LQS}, \mathrm{LIP}\}$ and $n \neq 4$, then every embedding (or immersion, respectively) of an (n-1)-dimensional $C$-manifold into an $n$-dimensional $C$-manifold without boundary can be relatively approximated by $C$-embeddings (or $C$-immersions, respectively). This was proved in [LT, Theorem 4.4] for the dimension pairs $(n, q)$ of the previous paragraph.

In Section 8 we show that if $M$ is an $n$-manifold, $n \neq 4$, and if $C \in\{$ LQC, LIP $\}$, every $C$-manifold structure on the boundary $\partial M$ of $M$ can be extended over $M$. This implies that every manifold carries a $C$-structure if every 4-manifold carries a $C$-structure.

In the last section we show that for every $n$, every LQC selfhomeomorphism of $R^{n}$ can be extended to an LQC selfhomeomorphism of $R_{+}^{n+1}$.

One of our starting-points was the paper $\left[\mathrm{G}_{2}\right]$ by Gauld, who applied [S]. We give both of his two theorems either as a corollary (3.2.2) or with a short proof (9.4).

Relatively little is known about the results of Sections 3, 4, and 5 for LQC or LIP in codimensions $\geqq 2$. The theorems of Section 3 are open in these codimensions; see, however, $[\mathrm{GV}]$ and $\left[\mathrm{G}_{1}\right]$ (in $\left[\mathrm{G}_{1}\right.$, Theorems 1 and 2] one can omit the assumption $n \neq 5$ using LIP straightening of TOP handles instead of PL straightening). In codimension two the equivalence and approximation theorems of Sections 4 and 5 do not hold as shown by the following facts: There are knotted locally PL flat embeddings $f: S^{n-2} \rightarrow R^{n} \quad(n \geqq 3)$ arbitrarily close to id, and there are embeddings $f: S^{1} \times S^{2 n-1} \rightarrow R^{2 n+2}(n \geqq 3)$ that cannot be approximated by PL embeddings or locally flat embeddings [M, Theorem 5.3] (here $S^{i}=\partial I^{i+1}$ ). However, by [Ve] every 
embedding of $I^{n}$ into a PL $(n+2)$-manifold can be approximated by locally flat PL embeddings. In codimensions $\geqq 3$ the results of Sections 4 and 5 are (essentially) known for TOP and PL (cf. [Mi], [Ma], [E]) but open for LQC and LIP. However, the author has partial results. The LQS and LIP approximation theorems of Section 7 are open in codimension two even in the absolute case.

One has considered $C$-embeddings and locally $C$-flat embeddings, $C \in\{\mathrm{LQS}$, LIP\}, separately. This is in order because of the following facts: In codimensions 0, 1,2 there are LIP embeddings which are not locally flat [LuV, Examples 3.10 (1) and (5)] and others which are locally flat but not locally LQC flat [ $T_{2}$, Section 17]. It is known that LIP embeddings in codimensions $\geqq 3$ are locally flat [LT, Theorem 1.14] but not whether they are locally LIP flat or at least locally LQC flat. In every codimension there are LQS embeddings which are not locally flat $\left[\mathrm{V}_{3}\right.$, Theorem 6.3] and others which are locally flat but not locally $L Q C$ flat $\left[\mathrm{V}_{3}\right.$, Remark 6.4.3].

Acknowledgements. I thank Robert Craggs and Thomas Price for giving me some details of certain ideas due to [Cr] and [PS], respectively.

\section{Terminology and preliminary results}

In this section we let $X$ and $Y$ denote metric spaces.

The letter $n$ denotes an integer which is positive except when $n$ is explicitly stated to be only non-negative. We identify $R^{n-1}$ with $\left\{x \in R^{n} \mid x_{n}=0\right\}$. We set $R_{+}^{n}=\left\{x \in R^{n} \mid x_{n} \geqq 0\right\}$, and for $n \geqq 0$ and $r>0, I^{n}=[-1,1]^{n}, I^{n}(r)=r I^{n}, J^{n}=(-1,1)^{n}$, $J^{n}(r)=r J^{n}, B^{n}(x, r)=\left\{y \in R^{n}:|x-y|<r\right\} \quad$ if $x \in R^{n}, B^{n}(r)=B^{n}(0, r), B^{n}(r)=\mathrm{cl} B^{n}(r)$, $B^{n}=B^{n}(1), S^{n}=\partial B^{n+1}$. If $A \subset R^{n}$ (and int $A \neq \emptyset$ ), we write $A_{+}=A \cap R_{+}^{n}$. The standard orthonormal basis of $R^{n}$ is written as $e_{1}, \ldots, e_{n}$. We set $I=[0,1]$ and $\mathbf{N}=\{1,2, \ldots\}$. We let id denote an inclusion map. Let $C_{+}(X)=\{f \mid f: X \rightarrow(0, \infty)$ continuous\}.

All manifolds are separable metric. Every metric, except the Euclidean one, is denoted by $d$. In a metric space, $d(A, B)$ denotes the distance between two sets $A, B$, with $d(\{x\}, B)=d(x, B)$, and $d(A)$ denotes the diameter of $A$.

Suppose that $f, g: X \rightarrow Y$ are continuous maps. We write

$$
d(f, g)=\sup \{d(f(x), g(x)) \mid x \in X\} .
$$

If $\varepsilon \in C_{+}(X)$, we let $U(f, \varepsilon)$ denote the set of all such $g$ that $d(f(x), g(x))<\varepsilon(x)$ for each $x \in X$. For some facts we need about these $\varepsilon$-neighborhoods $U(f, \varepsilon)$, we refer to $[\mathrm{LT}, 1.6]$.

If $A \subset X$, we let "near $A$ " mean "on an open neighborhood of $A$ ".

An isotopy $H$ of $X$ onto $Y$ is a continuous map $H: X \times I \rightarrow Y$ such that for each $t \in I$ the map $H_{t}: X \rightarrow Y, x \mapsto H(x, t)$, is a homeomorphism. As known, there are other definitions of an isotopy of $X$ onto $Y$, equivalent to this one if 
$X$ and $Y$ are locally compact. If $A \subset X$ and $H_{t}\left|A=H_{0}\right| A$ for each $t$, we say that $H$ is rel $A$. If $\varepsilon \in C_{+}(X)$ and $H_{t} \in U\left(H_{0}, \varepsilon\right)$ for each $t$, we say that $H$ is an $\varepsilon$-isotopy. If $X=Y$ and $H_{0}=\mathrm{id}$, we say that $H$ is an ambient isotopy of $X$. If $A, \varepsilon$ are as above, the $\varepsilon$-neighborhood of $A$ in $X$ is the set $N(A, \varepsilon)=$ $\{x \in X \mid \exists y \in A, d(x, y)<\varepsilon(y)\}$. If $H$ is an ambient $\varepsilon$-isotopy of $X$ rel $X \backslash N(A, \varepsilon)$, we say that the homeomorphism $H_{1}: X \rightarrow X$ is an $\varepsilon$-push of $(X, A)$. The combination of two isotopies $G$ and $H$ of $X$ onto $Y$ with $H_{0}=G_{1}$ is the isotopy $K$ of $X$ onto $Y$ defined by $K_{t}=G_{2 t}$ if $0 \leqq t \leqq 1 / 2$ and by $K_{t}=H_{2 t-1}$ if $1 / 2 \leqq t \leqq 1$. Given an isotopy $G$ of $X$ onto $Y$ and an ambient isotopy $H$ of $Y$, the isotopy $H * G$ of $X$ onto $Y$ is defined by $(H * G)_{t}=G_{2 t}$ if $0 \leqq t \leqq 1 / 2$ and by $(H * G)_{t}=$ $H_{2 t-1} G_{1}$ if $1 / 2 \leqq t \leqq 1 ; H * G$ is ambient if and only if $G$ is ambient.

We will often need the fact that if $U \subset X$ is open and $f: X \rightarrow Y, g: U \rightarrow Y$ are embeddings such that $d(f(x), g(x)) \leqq d(f(x), f[X \backslash U]) / 2$ for each $x \in U$, then $h=g \cup(f \mid X \backslash U): X \rightarrow Y$ is an embedding. This follows from

$$
\frac{1}{2} d(f(x), f(y)) \leqq d(h(x), h(y)) \leqq \frac{3}{2} d(f(x), f(y)) \text { for } \quad x \in U, \quad y \in X \backslash U .
$$

An immersion $f: X \rightarrow Y$ is a continuous map which embeds some neighborhood of each point of $X$.

An embedding $f: X \rightarrow Y$ is called ( $L$-) bilipschitz if, for some $L \geqq 1$,

$$
d(x, y) / L \leqq d(f(x), f(y)) \leqq L d(x, y) \text { for all } x, y \in X .
$$

If $f$ is only a function satisfying the right inequality, $f$ is called Lipschitz. A LIP map $f: X \rightarrow Y$ is one which is locally Lipschitz. Every PL map between polyhedra in Euclidean spaces is LIP. An embedding $f: X \rightarrow Y$ is called ( $\eta$-) quasisymmetric if, for some homeomorphism $\eta: R_{+}^{1} \rightarrow R_{+}^{1}$,

$$
d(f(a), f(x)) / d(f(b), f(x)) \leqq \eta(d(a, x) / d(b, x)) \text { for } \quad a, b, x \in X, \quad b \neq x .
$$

Like bilipschitz embeddings, quasisymmetric embeddings form a category; cf. $\left[\mathrm{TV}_{1}\right]$. Every bilipschitz embedding is quasisymmetric. An embedding $f: X \rightarrow Y$ is said to be a LIP embedding or an LQS embedding if $f$ is, locally, bilipschitz or quasisymmetric, respectively. Every LIP or LQS embedding of a compact space is bilipschitz or quasisymmetric [ $T V_{1}$, Theorem 2.23], respectively. For increasing embeddings $f: A \rightarrow R^{1}$, where $A \subset R^{1}$ is an interval, our definition of quasisymmetry is equivalent to the one in $\left[\mathrm{LV}_{2}, \mathrm{II} .7 .1\right]$. Hence, by $\left[\mathrm{LV}_{2}\right.$, II, Lemma 7.1 and (7.2)], every quasisymmetric homeomorphism $f:[0,1) \rightarrow[0,1)$ can be extended to a quasisymmetric homeomorphism $F: J^{1} \rightarrow J^{1}$ by reflection, i.e., if we set $F(x)=-f(-x)$ for $x<0$.

Let $n \geqq 2$, let $A \subset R^{n}$ be a set such that $A \subset \mathrm{cl}$ int $A$, and let $f: A \rightarrow R^{n}$ be an embedding. If there is $K \geqq 1$ such that the restrictions of $f$ to all components of int $A$ are $K$-quasiconformal in the sense of [ $\left.\mathrm{V}_{1}\right], f$ is called ( $K$-) quasiconformal. If each point of $A$ has an open neighborhood in $A$ on which $f$ is quasiconformal, 
$f$ is said to be LQC. The sets $A$ and the LQC embeddings $f: A \rightarrow R^{n}$ (observe that $f A \subset \mathrm{cl}$ int $f A$ ) form a category. We will freely use Theorem 35.1 of $\left[\mathrm{V}_{1}\right]$ about the piecewise construction of quasiconformal embeddings. In particular, an LQC embedding of a compact set is quasiconformal. Further, if $p: R^{n} \rightarrow R^{n}$ is the orthogonal reflection in $R^{n-1}$, we can extend every open quasiconformal (or LQC) embedding $f: U \rightarrow R_{+}^{n}$, where $U \subset R_{+}^{n}$ is open, to a quasiconformal (or LQC, respectively) embedding $F: U \cup p U \rightarrow R^{n}$ by reflection, i.e., by setting $F=p f p$ on $p U$; cf. $\left[\mathrm{V}_{1}\right.$, Theorem 35.2]. It follows from this and [ $\mathrm{V}_{3}$, Theorems 2.3 and 2.4] that if $U \subset R_{+}^{n}$ is open, an open embedding $f: U \rightarrow R_{+}^{n}$ is LQC if and only if $f$ is LQS. For embeddings $f: A \rightarrow R^{1}$, where $A \subset R^{1}$, we let "quasiconformal" and "LQC" mean "quasisymmetric" and "LQS", respectively. Also id: $R^{0} \rightarrow R^{0}$ is called LQC. It follows that if $n \geqq 1, U \subset R_{+}^{n}$ is open, and $f: U \rightarrow R_{+}^{n}$ is an open LQC embedding, $f \mid U \cap R^{n-1}: U \cap R^{n-1} \rightarrow R^{n-1}$ is LQC.

Let $C$ denote either LQS, LQC, or LIP. An atlas on an $n$-manifold, $n \geqq 0$, is called a $C$-atlas if its coordinate changes are $C$-homeomorphisms (cf. [LT, 1.3]). A $C$-structure is a maximal $C$-atlas, and a manifold equipped with a $C$-structure is called a $C$-manifold. Observe that LQC manifolds are the same as LQS manifolds. However, we prefer to use the term LQC rather than LQS whenever possible. We define $C$-embeddings of $C$-manifolds (of the same dimension if $C=\mathrm{LQC}$ ) by using charts. A $C$-immersion is a continuous map which is locally a $C$-embedding. The restrictions of the charts of the $C$-structure $\mathscr{A}$ of a $C$-manifold $M$ to the boundary $\partial M$ of $M$ form a $C$-atlas $\mathscr{A} \mid \partial M$ on $\partial M$. In fact, $\mathscr{A} \mid \partial M$ is a $C$-structure, because by $\left[\mathrm{TV}_{3}\right.$, Theorem 4.10 and Remark 4.11] every $C$-embedding $f: \partial N \rightarrow \partial Q$, where $N, Q$ are $n$-dimensional $C$-manifolds, can be extended to a $C$-embedding of a neighborhood of $\partial N$. If $M, Q$ are $C$-manifolds (of the same dimension in the case $C=\mathrm{LQC}), M$ is a subset of $Q$, and the inclusion $M \rightarrow Q$ is a $C$-embedding, then $M$ is called a $C$-submanifold of $Q$.

Let $C \in\{$ TOP, LQS, LQC, LIP $\}$ and let $Q$ be an $n$-dimensional $C$-manifold. Every discrete subspace of $Q$ is defined to be a locally $C$-flat 0 -submanifold of $Q$. For $1 \leqq k \leqq n$, we want every locally $C$-flat $k$-submanifold of $Q$ to meet $\partial Q$ transversally. For this purpose we introduce the notation $R_{++}^{n, k}=\left\{x \in R_{+}^{n} \mid x_{1}=\ldots=x_{n-k}=0\right.$, $\left.x_{n-1} \geqq 0\right\}$ for $k \geqq 2$ and $R_{++}^{n, 1}=\left\{x \in R_{+}^{n} \mid x_{1}=\ldots=x_{n-1}=0, x_{n} \in I\right\}$. We say that a subset $N$ of $Q$ is a locally $C$-flat $k$-submanifold of $Q(k \geqq 1)$ if for each point $x \in N$ there are an open neighborhood $U$ of $x$ in $Q$ and an open $C$-embedding $h: U \rightarrow R_{+}^{n}$ such that $h[U \cap N]=h U \cap R_{++}^{n, k}$. Since $R_{++}^{n, k}$ for $k \geqq 2$ is PL homeomorphic to $R_{+}^{k}$, it is easy to see that $N$ is then a $C$-manifold in a natural way (a $C$-submanifold of $Q$ except if $C=\mathrm{LQC}$ and $k \neq n)$. Clearly $N \cap \partial Q$ is a locally $C$-flat $(k-1)$-submanifold of $\partial Q$ and of $\partial N$. Let $M$ be a $k$-dimensional $C$-manifold and $f: M \rightarrow Q$ an embedding. If $k=0$, we call $f$ locally $C$-flat. For $k \geqq 1$, we say that $f$ is locally $C$-flat if for each $x \in M$ there are an open neighborhood $U \subset M$ of $x$, an open subset $V$ of $R_{+}^{n}$, a $C$-homeomorphism $h: U \rightarrow V \cap R_{++}^{n, k}$, and an open $C$-embedding $g: V \rightarrow Q$ such that $g \mid h U=f h^{-1}$. Then $f M$ is a locally $C$-flat sub- 
manifold of $Q$ and $f$ gives a $C$-homeomorphism $M \rightarrow f M$. Conversely, if $f M$ is a locally $C$-flat submanifold of $Q$ and $f$ gives a $C$-homeomorphism $M \rightarrow f M$, it is easy to see that $f$ is locally $C$-flat. An immersion $f: M \rightarrow Q$ is said to be locally $C$-flat if it is locally a locally $C$-flat embedding. We need the easily proved fact that if $M, Q$ are $C$-manifolds, $f: M \rightarrow Q$ is a locally $C$-flat embedding, and $N$ is a locally $C$-flat submanifold of $M$, then $f \mid N$ is locally $C$-flat, provided that either $f^{-1} \partial Q=\emptyset$ or $f^{-1} \partial Q=\partial M$. It is useful in this paper to have the concept of local LQS flatness, too, though it is equivalent to local LQC flatness.

Local PL flatness is defined in an analogous way. We need the fact that if $A$ is a closed subset of a PL manifold $M$ and $U \subset M$ is a neighborhood of $A$, there is a closed neighborhood $P$ of $A$ such that $P \subset U$ and $P$ is a locally PL flat PL submanifold of $M$. To construct such a $P$, choose a sufficiently fine triangulation $K$ of $M$, let $N(A, K)$ denote the union of all (closed) simplexes of $K$ which meet $A$, and set $P=N\left(N(A, K), K^{\prime \prime}\right)$, where $K^{\prime \prime}$ is the second barycentric subdivision of $K$; cf. [H, pp. 65, 67], [RS, pp. 32-35], [Ru, p. 20]. We also need the fact that a PL $n$-ball $B$ in $R_{+}^{n}$ is locally PL flat if $B \cap R^{n-1}$ is either empty [RS, Corollary 3.13] or a PL (n-1)-ball [H, Lemma 2.14].

The following lemma is needed in 3.1 .

2.1. Lemma. Let $I_{j}=I e_{j} \subset R^{3}, j=1,2,3$, let $T=I_{1} \cup I_{2} \cup I_{3}$, and let $f: T \rightarrow T$ be a homeomorphism.

(a) If $f \mid I_{i} \cup I_{j}$ is quasisymmetric for all $i, j, f$ is quasisymmetric.

(b) If $f \mid I_{1} \cup I_{2}$ and $f \mid I_{1} \cup I_{3}$ are quasisymmetric, $f$ is quasisymmetric.

(c) If $f \mid I_{1}$ is quasisymmetric, there is a quasisymmetric homeomorphism $F: T \rightarrow T$ such that $F=f$ on $I_{1} \cup\left\{e_{2}, e_{3}\right\}$ and $F$ is PL near $\left\{e_{2}, e_{3}\right\}$.

(d) If $f \mid I_{1} \cup I_{2}$ is quasisymmetric, there is a quasisymmetric homeomorphism $F: T \rightarrow T$ such that $F=f$ on $I_{1} \cup I_{2} \cup\left\{e_{3}\right\}$ and $F$ is PL near $e_{3}$.

Proof. We first observe that if $f \mid I_{1} \cup I_{2}$ is $\eta$-quasisymmetric and $H=\eta(1)$ $(\geqq 1)$, then, since $f(0)=0, \quad\left|f\left(x_{1}\right)\right| \leqq H\left|f\left(x_{2}\right)\right|$ and, hence, $\left|f\left(x_{1}\right)-f\left(x_{3}\right)\right| \leqq$ $H\left|f\left(x_{2}\right)-f\left(x_{3}\right)\right|$ whenever $x_{j} \in I_{j} \backslash 0,\left|x_{1}\right|=\left|x_{2}\right|$. By the aid of this fact it is easy to prove (a) and reduce (b) to (a) (we do not need (b)). To prove (c), we first extend $f \mid I_{1} \cup\left\{e_{2}, e_{3}\right\}$ to a homeomorphism $F_{0}: T \rightarrow T$ by setting $F_{0}\left(t e_{j}\right)=\left|f\left(t e_{1}\right)\right| f\left(e_{j}\right)$ for $t \in I, j=1,2,3$. By the reflection principle and (a), $F_{0}$ is quasisymmetric. The existence of $F$ now easily follows from the fact $\left(\left[\mathrm{LV}_{1}\right.\right.$, p. 13]) that every increasing quasisymmetric homeomorphism $\varphi: I \rightarrow I$ can be extended to a quasisymmetric homeomorphism $\varphi^{\prime}: 2 I \rightarrow 2 I$ which is PL near 2 . The proof of (d) is similar. 


\section{LQC or LIP approximation of homeomorphisms}

Consider the approximation result which is contained in $\left[\mathrm{TV}_{3}\right.$, Theorem 4.8] and which generalizes $\left[\mathrm{TV}_{3}\right.$, Theorem 4.4]. A slight modification of $\left[\mathrm{TV}_{3}\right.$, Remark 4.9] (where $s_{1}$ should have been chosen so that $g^{\prime}\left(s_{1}\right) \neq 0$ ) implies that this result also holds for $n=1$. Moreover, the condition " $f \mid \partial M$ is a $C$-embedding" can be replaced, as the proof shows, by the more general condition " $f \mid \partial M$ is a $C$-embedding near $B \cap \partial M$ ". In this section we apply this theorem to prove the more general Theorems 3.1 and 3.3, whose special case of empty $M_{1}$ and $M_{2}$ it is. In the proof of 3.3 we also use 4.1 .

3.1. Theorem. Let $C \in\{\mathrm{LQC}, \mathrm{LIP}\}$, let $Q_{1}, Q_{2}$ be $n$-dimensional $C$-manifolds, let $A, B \subset Q_{1}$ be closed and $B^{\prime} \subset Q_{1}$ a neighborhood of $B$, let $M_{i}$ be a closed locally $C$-flat $(n-1)$-submanifold of $Q_{i}, i=1,2$, such that $\left(M_{1} \backslash A\right) \cap \partial Q_{1}=$ $\partial M_{1} \backslash A$, let $f: Q_{1} \rightarrow Q_{2}$ be a homeomorphism such that $f$ is a $C$-embedding near $A$ and $f M_{1}=M_{2}$, and let $\varepsilon \in C_{+}\left(Q_{1}\right)$.

(a) Provided $n \neq 4,5,6$, there is an $\varepsilon$-isotopy $H$ of $Q_{1}$ onto $Q_{2}$ such that

(1) $H_{0}=f$,

(2) $H_{t}=f$ for each $t \in I$ near $A$ and on $Q_{1} \backslash B^{\prime}$,

(3) $H_{t} M_{1}=M_{2}$ for each $t \in I$,

(4) $H_{1}$ is a $C$-embedding near $A \cup B$.

(b) If $X \in\left\{\partial M_{1}, \partial Q_{1}, M_{1}\right\}$ and if $f \mid X$ is a $C$-embedding near $B \cap X$, then (a) is also true for $n=6$, and $H$ can be chosen to be $\operatorname{rel} X$.

(c) Suppose that $f \mid X$ is a $C$-embedding near $B \cap X$ for each $X \in\left\{\partial Q_{1}, M_{1}\right\}$. If $C=\mathrm{LQC}$ and $n=2$, suppose in addition that $f \mid \partial Q_{1} \cup M_{1}$ is LQS near $\partial M_{1} \cap$ $(B \backslash A)$. Then (a) is also true for $n=5$ or 6 , and $H$ can be chosen to be rel $\partial Q_{1} \cup M_{1}$.

Proof. Note that $f \mid \partial M_{1}$ is necessarily a $C$-embedding near $B \cap \partial M_{1}$ in (b) and (c). As in the proof of [ $\mathrm{TV}_{3}$, Theorem 4.4], the general case can be reduced to the special case where $B$ is compact and either $\left(Q_{1}, M_{1}\right)=\left(Q_{2}, M_{2}\right) \in\left\{\left(R^{1}, \emptyset\right)\right.$, $\left.\left(R_{+}^{1}, \emptyset\right),\left(R^{1},\{0\}\right)\right\}$ if $n=1$ or, if $n \geqq 2, Q_{1}, Q_{2}$ are open subsets of $R_{+}^{n}$ and $M_{i}=$ $Q_{i} \cap L_{0}, i=1,2$, where $L_{0}=R^{n-2} \times\{0\} \times R_{+}^{1} \subset R_{+}^{n}$. For $n=1$, the case $\left(Q_{i}, M_{i}\right)=$ $\left(R^{1}, \emptyset\right), i=1,2$, is proved in $\left[\mathrm{TV}_{3}\right.$, Remark 4.9]; the remaining cases can be proved similarly.

Let now $n \geqq 2$. To prove the special case, we may additionally assume that $B^{\prime}$ is compact and $Q_{1}$ connected. Choose an open neighborhood $A^{\prime}$ of $A$ such that $f \mid A^{\prime}$ is a $C$-embedding. Let $Q_{i}^{j}=Q_{i} \cap\left(R^{n-2} \times j R_{+}^{1} \times R_{+}^{1}\right), j=-1,1$. Then $Q_{i}=Q_{i}^{1} \cup Q_{i}^{-1}$ and $Q_{i}^{1} \cap Q_{i}^{-1}=M_{i}$. It is easy to see that $f Q_{1}^{1}$ is either $Q_{2}^{1}$ or $Q_{2}^{-1}$. Hence we may assume that $f Q_{1}^{j}=Q_{2}^{j}, j=-1,1$. Let $L_{j}=R^{n-2} \times j R_{+}^{1} \times\{0\} \subset R_{+}^{n}$, $j=-1,1$. Then $f\left[Q_{1} \cap L_{j}\right]=Q_{2} \cap L_{j}$. Note that there is a PL homeomorphism of $R^{n-2} \times j R_{+}^{1} \times R_{+}^{1}$ onto $R_{+}^{n}$ which is the identity on $L_{j}, j=-1,1$.

By Theorem 4.8 of $\left[\mathrm{TV}_{3}\right]$ and its proof, for all $j=-1,0,1$ and $\delta>0$ there is a $\delta$-isotopy $G^{j}$ of $Q_{1} \cap L_{j}$ onto $Q_{2} \cap L_{j}$ with the following properties: $G_{0}^{j}=$ 
$f \mid Q_{1} \cap L_{j}, G^{j}$ is rel $\left(Q_{1} \cap L_{j}\right) \backslash D$ for some compact neighborhood $D$ (independent of $j$ ) of $B \backslash A^{\prime}$ in int $_{Q_{1}} B^{\prime} \backslash A$, and $G_{1}^{j}$ is a $C$-embedding near $B \cap L_{j} ; G_{t}^{1}=$ $G_{t}^{0}=G_{t}^{-1}$ on $\partial M_{1}$ for each $t \in I ; G^{j}$ is rel $\partial M_{1}$ whenever $f \mid \partial M_{1}$ is a $C$-embedding near $B \cap \partial M_{1}$, and finally, $G^{j}$ is rel $X \cap L_{j}$ whenever $X \in\left\{\partial Q_{1}, M_{1}\right\}$ and $f \mid X$ is a $C$-embedding near $B \cap X$. In the part (a) we needed $n \neq 5,6$, in the part (b) $n \neq 5$. For $j=-1,1$ define an isotopy $K^{j}=G^{0} \cup G^{j}$ of $\partial Q_{1}^{j}=Q_{1} \cap\left(L_{0} \cup L_{j}\right)$ onto $\partial Q_{2}^{j}$. Then $K_{1}^{j}$ is a $C$-embedding near $B \cap \partial Q_{1}^{j}$ if $(C, n) \neq(\mathrm{LQC}, 2)$. To obtain this claim for $(C, n)=(\mathrm{LQC}, 2)$, too, additional care is needed. We may assume that $\{0\}=\partial M_{1} \subset B \backslash A$. The part (c) is clear, since $K_{1}^{j}=f \mid \partial Q_{1}^{j}$ is LQS near 0 by our particular assumption. In the part (a), $\left[\mathrm{TV}_{3}\right.$, Remark 4.9] shows that the isotopies $G^{i}, i=-1,0,1$, can be chosen in such a way that $G_{1}^{i}$ is PL near 0 , in which case the claim holds. Consider the subcase of (b) where $f \mid M_{1}$ is LQS near $B \cap M_{1}$ (implying $G_{1}^{0}=f \mid M_{1}$ ). We may assume that $G_{1}^{1} \cup G_{1}^{-1}$ is PL near 0 . Hence, modifying $G^{1} \cup G^{-1}$ by combining it with a new isotopy, we may assume by 2.1 (c) that $G_{1}^{1} \cup G_{1}^{0} \cup G_{1}^{-1}$ is LQS near 0 , whence the claim. Finally, the treatment of the subcase of (b) where $f \mid \partial Q_{1}$ is LQS near $B \cap \partial Q_{1}$ is similar; we now use 2.1(d).

Again by Theorem 4.8 of $\left[\mathrm{TV}_{3}\right]$ and its proof, if the above $\delta$ is sufficiently small, for each $j=-1,1$ there is an $\left(\varepsilon \mid Q_{1}^{j}\right)$-isotopy $H^{j}$ of $Q_{1}^{j}$ onto $Q_{2}^{j}$ which extends $K^{j}$ such that $H_{0}^{j}=f \mid Q_{1}^{j}, H_{t}^{j}=f$ for each $t \in I$ near $A \cap Q_{1}^{j}$ and on $Q_{1}^{j} \backslash B^{\prime}$, and $H_{1}^{j}$ is a $C$-embedding near $B \cap Q_{1}^{j}$. Then $H=H^{1} \cup H^{-1}$ is the desired isotopy.

3.2. Remarks. 1 . In the case $n=1$, Theorem 3.1 also holds if we omit the assumption $\left(M_{1} \backslash A\right) \cap \partial Q_{1}=\partial M_{1} \backslash A(=\emptyset)$. In fact, the isotopy $H$ given by 3.1 with $M_{i}$ replaced by $M_{i} \backslash \partial Q_{i}$ is then the desired isotopy, since it is rel $\partial Q_{1} \cup M_{1}$.

2. The following special case of 3.1 is $\left[\mathrm{G}_{2}\right.$, Theorem 1]: If $C \in\{\mathrm{LQC}, \mathrm{LIP}\}$, $n \neq 4,5$, and $M_{1}, M_{2}$ are compact locally $C$-flat $(n-1)$-submanifolds of $S^{n}$ without boundary such that $\left(S^{n}, M_{1}\right)$ is homeomorphic to $\left(S^{n}, M_{2}\right)$, then $\left(S^{n}, M_{1}\right)$ is $C$-homeomorphic to $\left(S^{n}, M_{2}\right)$.

In the case $\partial M_{1} \subset A$, the next theorem also follows from 3.1.

3.3. Theorem. Let $C, Q_{i}, A, B, B^{\prime}, M_{i}, f, \varepsilon$ be as in 3.1 but such that $M_{1} \subset$ $A \cup B$ and that the assumption $\left(M_{1} \backslash A\right) \cap \partial Q_{1}=\partial M_{1} \backslash A$ is replaced by the assumption $M_{1} \backslash A \subset$ int $Q_{1}$.

(a) The part (a) of 3.1 holds with (3) replaced by the condition $H_{1} M_{1}=M_{2}$.

(b) If $X \in\left\{\partial M_{1}, M_{1}\right\}$ and if $f \mid X$ is a C-embedding, then (a) is also true for $n=6$, and $H$ can be chosen such that $H_{1}|X=f| X$.

(c) If $f \mid \partial Q_{1}$ is a C-embedding near $B \cap \partial Q_{1}$, then (b) with $X=M_{1}$ is also true for $n=5$ or 6 , and $H$ can be chosen to be rel $\partial Q_{1}$ in (a) and (b).

Proof. Choose open sets $A_{1}, A_{2}, B_{1} \subset Q_{1}$ such that $A \subset A_{2} \subset \bar{A}_{2} \subset A_{1}, f$ is a $C$-embedding near $\bar{A}_{1}, B \subset B_{1} \subset \bar{B}_{1} \subset$ int $_{Q_{1}} B^{\prime}$, and $f \mid \partial Q_{1}$ is a $C$-embedding near $\bar{B}_{1} \cap \partial Q_{1}$ in (c). Then $N=f\left[B^{\wedge} \backslash A_{2}\right] \cap$ int $Q_{2}$ is a neighborhood of $f\left[M_{1} \backslash A_{1}\right]$ 
in $Q_{2}$. Choose $\eta \in C_{+}\left(Q_{2}\right)$ with $U\left(\operatorname{id}_{Q_{2}}, \eta\right) \circ U(f, \eta f) \subset U(f, \varepsilon)$. Then choose $\eta_{1} \in C_{+}\left(Q_{1}\right), \eta_{1} \leqq \eta f$, such that $\delta=\eta_{1} \mid M_{1}$ is the function given by 4.1 with the substitution $C \mapsto C, n \mapsto n, M \mapsto M_{1}, Q \mapsto Q_{2}, A, B \mapsto M_{1}, U \mapsto A_{1} \cap M_{1}, f \mapsto f \mid M_{1}, N \mapsto N$, $\varepsilon \mapsto \eta \mid N$. By [TV $\mathrm{TV}_{3}$, Theorem 4.8] there is an $\eta_{1}$-isotopy $G$ of $Q_{1}$ onto $Q_{2}$ rel $A_{1} \cup$ $\left(Q_{1} \backslash B^{\prime}\right)$ such that $G_{0}=f, G_{1} \mid A_{1} \cup B_{1}$ is a $C$-embedding, and $G$ is rel $\partial Q_{1}$ in (c). Then $f_{1}=G_{1} \mid M_{1}$ is a locally $C$-flat embedding in $U\left(f \mid M_{1}, \delta\right)$ with $f_{1}=f$ on $A_{1} \cap M_{1}$. Again by $\left[\mathrm{TV}_{3}\right.$, Theorem 4.8], there is a $C$-homeomorphism $f_{2}: M_{1} \rightarrow M_{2}$ in $U\left(f \mid M_{1}, \delta\right)$ such that $f_{2}=f$ on $A_{1} \cap M_{1}$ and that $f_{2}|X=f| X$ whenever $X \in\left\{\partial M_{1}, M_{1}\right\}$ and $f \mid X$ is a $C$-embedding. Then $f_{2}$ is locally $C$-flat as an embedding into $Q_{2}$. Hence, by the definition of $\delta$, there is an ambient $\eta$-isotopy $K$ of $Q_{2}$ rel $Q_{2} \backslash N$ such that $K_{1}$ is a $C$-homeomorphism and $K_{1} f_{1}=f_{2}$. It follows that $H=K * G$ is the desired isotopy.

3.4. Remark. For $n=2$, the modification of 3.3 holds where the assumption $M_{1} \backslash A \subset \operatorname{int} Q_{1}$ and the assertion (c) are omitted and where $H_{1}\left|\partial M_{1}=f\right| \partial M_{1}$. This result can be proved as 3.3 by the aid of 4.2 .

3.5. Example. For every $C \in\{\mathrm{LQC}, \mathrm{LIP}\}$ and for every $n \geqq 1$, each of the parts (a), (b), and (c) of 3.1 and of 3.3 is false without the assumption that $M_{i}$ is closed in $Q_{i}$. We show this by constructing a locally LIP flat $(n-1)$-submanifold $M$ of $R^{n}$ without boundary and a homeomorphism $f: R^{n} \rightarrow R^{n}$ which is a LIP embedding near $M$ such that $f M$ is not the image of $M$ under any LQC homeomorphism $h: R^{n} \rightarrow R^{n}$. Suppose first $n \geqq 2$. We modify [ $\mathrm{V}_{3}$, Example 5.11]. Let $M=R^{n-1} \backslash 0$. Set $\varphi(t)=t \sin (1 / t)$ for $t>0$ and $\varphi(0)=0$, and define $f(x, t)=$ $(x, t+\varphi(|x|))$ for $x \in R^{n-1}, t \in R^{1}$. If now $h: R^{n} \rightarrow R^{n}$ were an LQC homeomorphism such that $h M=f M$, then $h R^{n-1}=f R^{n-1}$, which is impossible, since no neighborhood of the origin in $f R^{n-1}$ is of bounded turning; cf. [TV Consider $n=1$. We let $M=\{ \pm 1 / j \mid j \in \mathbf{N}\}$ and define $f(x)=x^{2}$ for $x \geqq 0$ and $f(x)=x$ for $x<0$. Suppose that there is an LQS homeomorphism $h: R^{1} \rightarrow R^{1}$ such that $h M=f M$. We may assume that $h$ is increasing. Then $h|\bar{M}=f| \bar{M}$, which is impossible, since $f \mid \bar{M}$ is not locally quasisymmetric at the origin.

\section{Equivalence of close locally $C$-flat embeddings}

In this section we prove the following two theorems. For a discussion on them, see 4.3.

4.1. Theorem. Let $C \in\{$ TOP, LQC, LIP $\}$ and $n \neq 4$, let $M$ be an (n-1)and $Q$ an n-dimensional $C$-manifold, let $A \subset M$ be closed, $B \subset M$ a neighborhood of $A$, and $U \subset M$ open, let $f: M \rightarrow Q$ be an embedding with $f[A \backslash U] \subset \operatorname{int} Q$, let $N \subset Q$ be a neighborhood of $f[A \backslash U]$, and let $\varepsilon \in C_{+}(N)$. If $C \neq \mathrm{TOP}$ and $n \geqq 2$, suppose that $f[A \backslash U]$ is closed in $Q$. Then there is $\delta \in C_{+}(M)$ with the 
following property: If $f_{1}, f_{2}: M \rightarrow Q$ are locally $C$-flat embeddings such that $d\left(f_{i}(x), f(x)\right)<\delta(x)$ for each $x \in B, i=1,2$, and $f_{1}\left|U=f_{2}\right| U$, there is an ambient isotopy $H$ of $Q$ rel $Q \backslash N$ such that $d\left(H_{t}(x), x\right)<\varepsilon(x)$ for all $x \in N$ and $t \in I$, $H_{t}$ is a C-homeomorphism for each $t \in I$, and $H_{1} f_{1}=f_{2}$ on $N(A, \delta)$.

4.2. Theorem. For $n \leqq 3$, Theorem 4.1 also holds with the following modifications: 1) The assumption $f[A \backslash U] \subset \operatorname{int} Q$ is replaced by the following assumption. Let $T \subset M$ be an open neighborhood of $A \backslash U$. Suppose that $T \cap f^{-1} \partial Q$ is an $(n-2)$-submanifold of $\partial T$ whenever either $n=2$ or $C=\mathrm{TOP}, n=3$ and that $T \cap f^{-1} \partial Q=\partial T$ whenever $C \neq \mathrm{TOP}, n=3$. 2) The embeddings $f_{1}, f_{2}$ are assumed to have the additional property that either $T \cap f_{i}^{-1} \partial Q=T \cap f^{-1} \partial Q, i=1,2$, whenever $n \geqq 2$ or $T \cap f_{1}^{-1} \partial Q=T \cap f_{2}^{-1} \partial Q$ whenever $n=1$. 3) Only $H_{1}$ is claimed to be a $C$-homeomorphism, not $H_{t}$ for each $t \in I$.

4.3. Remarks. 1 . For $C=\mathrm{TOP}$ and $n \leqq 3,4.2$ generalizes 4.1 .

2. The forms of 4.1 and 4.2 where $N$ is more generally a neighborhood of $f\left[A \backslash\right.$ int $\left._{M} \bar{U}\right]$ only follow trivially.

3. Consider the (uninteresting) case $n=1$. In 4.2 the isotopy $H$ can, as in 4.1, be chosen such that $H_{t}$ is a $C$-homeomorphism for each $t \in I$ also if $C \neq \mathrm{TOP}$. We omit the proofs of 4.1 and 4.2 because they differ from the proofs in the case $n \geqq 2$. We only remark that these results reduce to the special case of 4.2 where $C=$ LIP, $A=M, U=\emptyset$, for which there exists an easy direct proof.

4. For all $C \neq$ TOP and $n \geqq 2$, the assumption in 4.1 and 4.2 that $f[A \backslash U]$ is closed is essential. For example, for every $\delta \in C_{+}\left(B^{n-1}\right)$, where $n \geqq 2$, the $\delta$-neighborhood of the inclusion $B^{n-1} \rightarrow R^{n}$ contains a locally LIP flat embedding which is not quasisymmetric, and which thus is not a restriction of any LQC homeomorphism $R^{n} \rightarrow R^{n}$.

5. For $C=$ TOP, 4.1 and 4.2 are known in the absolute case $A=M, U=\emptyset$ (we may now assume that $f$ is closed). For $n \geqq 5$ this is due to Price and Seebeck [PS, Theorem 3]. The case $n=3$ follows from Craggs [Cr, Theorem 8.2] (cf. [ $\mathrm{B}_{1}$, Theorem 9]); this result of [Cr] also implies for $n=2$ the absolute case of 4.1. Of course, 4.2 for $C=$ TOP, $n=2$ is well-known, but it seems that it does not exist in the literature.

6. The first of the three stages of the proofs of 4.1 and 4.2 consists of handle lemmas. For $C=$ TOP these lemmas base on the handle lemma [PS, Theorem 2] if $n \geqq 5$, on [Cr, Theorem 8.1] if $n=3$ (cf. the paragraph just before Theorem 8.2 in [Cr]), and on the Schoenflies theorem if $n=2$. The handle lemmas for $C \neq \mathrm{TOP}$ follow from the TOP handle lemmas and 3.1.

We begin with $C=\mathrm{TOP}, n=3$.

4.4. Lemma. Let $M$ be a PL 2-manifold, $Q$ a PL 3-manifold, $f: M \rightarrow Q$ a closed locally flat embedding, $A \subset Q$ closed with $f$ being PL near $f^{-1} A$, and $\varepsilon \in C_{+}(Q \backslash A)$. Then there is an ambient isotopy $H$ of $Q$ rel $A$ such that $d\left(H_{t}(x), x\right)<$ $\varepsilon(x)$ for all $x \in Q \backslash A$ and $t \in I$ and that $H_{1} f$ is PL. 
Proof. Choose a closed polyhedral neighborhood $N$ of $A$ in $Q$ such that $f$ is PL near $f^{-1} N$. Obviously, there is a triangulation $K$ of the space $S=f M \cup N$ such that $f$ is $\mathrm{PL}, N$ a polyhedron, and the inclusion map $N \rightarrow Q$ PL with respect to $K$. Then $S_{0}=S \cap \partial Q$ is a polyhedron under $K$. We may assume that a subcomplex $K_{0}$ of $K$ triangulates $S_{0}$. We conclude, by the analogue of [ $\mathrm{B}_{1}$, Theorem 8'] for 2-manifolds without boundary (this is obviously true) or directly, that a subdivision of $K_{0}$, which we may assume to be $K_{0}$ itself, can be extended to a triangulation $K^{\prime}$ of the space $\partial Q$. Then by $\left[\mathrm{B}_{1}\right.$, Theorem $\left.8^{\prime}\right]$ a subdivision of the triangulation $K \cup K^{\prime}$ of $S \cup \partial Q$, which we may assume to be $K \cup K^{\prime}$ itself, can be extended to a triangulation $K_{1}$ of the space $Q$. By [ $\mathrm{B}_{1}$, Theorem 4], for every $\delta \in C_{+}(Q \backslash A)$ there is a PL homeomorphism $g:\left(Q, K_{1}\right) \rightarrow Q$ such that $g \mid A=\mathrm{id}$ and $d(g(x), x)<\delta(x)$ for each $x \in Q \backslash A$. Then $g f$ is PL. If $\delta$ is small enough, by [Ks, Theorem] there is an ambient isotopy $H$ of $Q \operatorname{rel} A$ such that $H$ is an $\varepsilon$-isotopy on $Q \backslash A$ and $H_{1}=g$.

\subsection{Lemma. Theorem 4.2 holds if $C=\mathrm{TOP}, n=3$, and $A=B=T=M$.}

Proof. We first reduce the lemma to the case where $f$ is closed. Since $f M$ is locally compact, it has an open neighborhood $Q_{0}$ in $Q$ where it is closed. We may assume that $N$ is open, $N \subset Q_{0}$, and $\varepsilon(x) \leqq d(x, Q \backslash N) / 2$ for each $x \in N$. Let $\delta$ with $\delta(x) \leqq d\left(f(x), Q \backslash Q_{0}\right)$ for each $x \in M$ be the function given by the lemma if $Q$ is replaced by $Q_{0}$. Consider $f_{1}, f_{2}$ as in the lemma; then $f_{i} M \subset Q_{0}$. Let $H^{0}$ be the ambient isotopy of $Q_{0}$ thus obtained. Extending $H_{t}^{0}$ for each $t \in I$ by the identity of $Q \backslash Q_{0}$ we get the desired ambient isotopy $H$ of $Q$.

Thus we may assume that $f$ is closed. Then we may also assume that there is $\varepsilon^{\prime} \in C_{+}(Q)$ extending $\varepsilon$. We may further assume that $N$ is open and that $M$ and $Q$ are PL manifolds. Then $L=f^{-1} \partial Q$ is a PL 1 -submanifold of $\partial M$. Choose closed locally PL flat submanifolds $M_{1}, M_{2} \subset U$ of $M$ such that $M_{1}$ is a neighborhood of $M \backslash f^{-1} N, M_{2}$ is a neighborhood of $M_{1}$, and $M_{2} \cap L$ is a 1-manifold. Choose an open set $N_{1} \subset Q$ such that $\bar{N}_{1} \subset N$ and

$$
f \overline{M \backslash M_{2}} \subset N_{1}, \quad f M_{1} \cap \bar{N}_{1}=\emptyset .
$$

Then choose an open neighborhood $N_{2}$ of $\bar{N}_{1} \cup f \overline{M \backslash M_{1}}$ in $Q$ with $\bar{N}_{2} \subset N$. Choose $\eta_{0} \in C_{+}(Q)$ such that if $\varphi_{1}, \ldots, \varphi_{5}: Q \rightarrow Q$ are homeomorphisms in $U\left(\mathrm{id}, \eta_{0}\right)$, then $\varphi_{1}^{-1} \varphi_{2}^{-1} \varphi_{3} \varphi_{4} \varphi_{5} \in U\left(\mathrm{id}, \varepsilon^{\prime}\right)$. Define a continuous $\mu: Q \rightarrow R_{+}^{1}$ by $\mu(x)=$ $\min \left(\eta_{0}(x) / 2, d\left(x, Q \backslash N_{2}\right)\right)$. Let $v \in C_{+}(M)$ be the function given by [Cr, Theorem 8.1] with the substitution $M \mapsto Q, K \mapsto M, K_{a} \mapsto L, L \mapsto M_{1}, f \mapsto f, \mu \mapsto \mu$. That is, if $g_{1}, g_{2}: M \rightarrow Q$ are PL embeddings in $U(f, v)$ such that $g_{1}=g_{2}$ on $M_{1}$ and $g_{i}^{-1} \partial Q=L$, there is an ambient (PL) isotopy $G$ of $Q$ such that $G_{1} g_{1}=g_{2}$ and $d\left(G_{t}(x), x\right) \leqq \mu(x)$ for all $x \in Q$ and $t \in I$. Choose $\delta \in C_{+}(M)$ and $\eta \in C_{+}(Q)$, $\eta \leqq \eta_{0}$, such that if $\varphi \in U(f, \delta)$ is an embedding and $\varphi_{1}, \varphi_{2} \in U\left(\mathrm{id}_{Q}, \eta\right)$, then $\varphi$ is closed, $\varphi_{1}[Q \backslash N] \subset Q \backslash N_{2}$, (4.6) holds with $f$ replaced by $\varphi_{1} \varphi$, and $\varphi_{2} \varphi_{1} \varphi \epsilon$ $U(f, v)$. 
We show that this $\delta$ is the desired function. Let $f_{1}, f_{2}$ be as in 4.5. Then, since $\left(f_{i} \mid M_{2}\right)^{-1} \partial Q=M_{2} \cap L$ is a 1-submanifold of $\partial M_{2}$, the embedding $f_{1} \mid M_{2}=$ $f_{2} \mid M_{2}$ is locally flat, and, hence, by 4.4 there is a homeomorphism $g: Q \rightarrow Q$ in $U$ (id, $\eta$ ) such that $f_{i}^{\prime}=g f_{i}, i=1,2$, is PL on $M_{2}$. Since $\left(f_{i}^{\prime}\right)^{-1}\left[Q \backslash N_{1}\right] \subset \operatorname{int}_{M} M_{2}$, Lemma 4.4 gives an ambient $\eta$-isotopy $G^{i}$ of $Q$ rel $Q \backslash N_{1}$ such that $g_{i}=G_{1}^{i} f_{i}^{\prime}$ is PL. We have $g_{i} \in U(f, v), g_{1}\left|M_{1}=g_{2}\right| M_{1}$, and $g_{i}^{-1} \partial Q=L$. Thus the second paragraph of the proof gives a certain isotopy $G$. Then by defining $H_{t}=$ $g^{-1}\left(G_{t}^{2}\right)^{-1} G_{t} G_{t}^{1} g$ for $t \in I$ we obtain an ambient $\varepsilon^{\prime}$-isotopy $H$ of $Q$ rel $Q \backslash N$ such that $H_{1} f_{1}=f_{2}$.

The next lemma is needed for $n=2$.

4.7. Lemma. Let $N \subset R^{2}$ be a neighborhood of $I$ and let $\varepsilon>0$. Then there is $\delta>0$ with the following property: If $f_{1}, f_{2}:[-1,2] \rightarrow R^{2}$ are embeddings with $d\left(f_{i}, \mathrm{id}\right)<\delta$, there is a homeomorphism $h: R^{2} \rightarrow R^{2}$ such that $d(h, \mathrm{id})<\varepsilon, h \mid R^{2} \backslash N=\mathrm{id}$, and $h f_{1}=f_{2}$ on I. Moreover, if

$$
f_{1}=f_{2} \quad \text { on } \quad[-1,2] \backslash I,
$$

$h$ can be chosen such that $h f_{1}=f_{2}$ on $[-1,2]$.

Proof. Choose an integer $p \geqq 2$ such that if $\eta=1 / p$, then $\eta<\varepsilon / 8$ and $(-2 \eta, 1+2 \eta) \times(-\eta, \eta) \subset N$. Let $\delta=\eta / 4$. Let $f_{1}, f_{2}:[-1,2] \rightarrow R^{2}$ be embeddings with $d\left(f_{i}, \mathrm{id}\right)<\delta$. Choose arcs $A_{i j k}, i \in\{1,2\}, j \in\{-1,0, \ldots, p+1\}, k \in\{0,1\}$, with endpoints $f_{i}(j \eta)$ and $\left(j \eta,(-1)^{k} \eta\right)$ such that

$$
\begin{aligned}
& \operatorname{int} A_{i j k} \subset((j-1 / 2) \eta,(j+1 / 2) \eta) \times(-\eta, \eta) \backslash f_{i}[-1,2], \\
& A_{1 j k}=A_{2 j k} \text { for } j \in\{-1, p+1\} \text { in the case (4.8). }
\end{aligned}
$$

Then the open arcs int $A_{i j k}$ are disjoint. The arcs $A_{i j k}$ can be found, for example, as follows. Let $f_{i}\left(a_{j k}\right)$ be the point of $f_{i}[-1,2] \cap\{j \eta\} \times[-\eta, \eta]$ closest to $\left(j \eta,(-1)^{k} \eta\right)$. We have $\left|a_{j k}-j \eta\right|<\delta$ and $f_{i} J_{j k} \subset(j \eta-2 \delta$, ji $+2 \delta) \times(-\delta, \delta)$, where $J_{j k}=\left[a_{j k}, j \eta\right]$ (or $\left.\left[j \eta, a_{j k}\right]\right)$. The arc $A_{i j k}$ is now the union of a subarc of the segment $\{j \eta\} \times[-\eta, \eta]$ and an arc close to $f_{i} J_{j k}$.

There are Jordan domains

$$
G_{i j k} \subset((j-3 / 2) \eta, \quad(j+1 / 2) \eta) \times(-\eta, \eta)
$$

for $i \in\{1,2\}, j \in\{0, \ldots, p+1\}, k \in\{0,1\}$ such that

$$
\partial G_{i j k}=[(j-1) \eta, j \eta] \times\left\{(-1)^{k} \eta\right\} \cup A_{i(j-1) k} \cup f_{i}[(j-1) \eta, j \eta] \cup A_{i j k},
$$

if (4.8) holds or if $j \notin\{0, p+1\}$, or such that

$$
\begin{gathered}
G_{i j 0}=G_{i j 1} \text { for } j \in\{0, p+1\}, \\
\partial G_{i 00}=[-\eta, 0] \times\{-\eta, \eta\} \cup\{-\eta\} \times[-\eta, \eta] \cup A_{i 00} \cup A_{i 01}, \\
\partial G_{i(p+1) 0}=[1,1+\eta] \times\{-\eta, \eta\} \cup\{1+\eta\} \times[-\eta, \eta] \cup A_{i p 0} \cup A_{i p 1},
\end{gathered}
$$

if (4.8) does not hold. 
By the Schoenflies theorem there is a homeomorphism $h: R^{2} \rightarrow R^{2}$ such that

$$
\begin{gathered}
h \bar{G}_{1 j k}=\bar{G}_{2 j k} \text { for } j \in\{0, \ldots, p+1\}, \quad k \in\{0,1\}, \\
h=\text { id on } R^{2} \backslash \operatorname{int}\left(\cup\left\{\bar{G}_{1 j k} \mid j \in\{0, \ldots, p+1\}, k \in\{0,1\}\right\}\right), \\
h f_{1}=f_{2} \text { on } I, \\
h f_{1}=f_{2} \text { on }[-1,2] \text { in the case (4.8). }
\end{gathered}
$$

Then $h \mid R^{2} \backslash N=$ id. Finally, since $\bar{G}_{1 j k} \cap \bar{G}_{2 j k} \neq \emptyset$ and $d\left(\bar{G}_{i j k}\right)<\varepsilon / 2$ for all $i, j, k$, we have $d(h, \mathrm{id})<\varepsilon$.

The next two lemmas are the handle lemmas needed for 4.1.

4.9. Lemma. Let $C \in\{\mathrm{TOP}, \mathrm{LQC}, \mathrm{LIP}\}$, let $n \neq 4$, let $k, m \geqq 0$ be integers with $k+m=n-1$, let $f: I^{n-1} \rightarrow R^{n}$ be an embedding, and let $\varepsilon>0$. Then there is $\delta>0$ with the following property: If $f_{1}, f_{2}: I^{n-1} \rightarrow R^{n}$ are locally $C$-flat embeddings such that $d\left(f_{i}, f\right)<\delta$ and $f_{1}=f_{2}$ on $\left(I^{k} \backslash I^{k}(1 / 2)\right) \times I^{m}$, there is an ambient $\varepsilon$-isotopy $H$ of $R^{n}$ rel $R^{n} \backslash N\left(f I^{n-1}(1 / 2), \varepsilon\right)$ such that $H_{t}$ is a $C$-homeomorphism for each $t \in I$ and $H_{1} f_{1}=f_{2}$ on $I^{k} \times I^{m}(1 / 2)$.

Proof. The case $n=1$ being trivial, we may assume $n \geqq 2$. Consider first $C=$ TOP. The case $n \geqq 5$ is [PS, Theorem 2]. The case $n=3$ follows from 4.5 (apply it to $f \mid I^{k} \times I^{m}(1 / 2)$ ). Let now $n=2$. Since $f$ can be extended to a homeomorphism $R^{2} \rightarrow R^{2}$, we may assume that $f$ is the inclusion map. Then 4.7 and Alexander's construction of isotopies (cf. [RS, Proposition 3.22]) give the lemma.

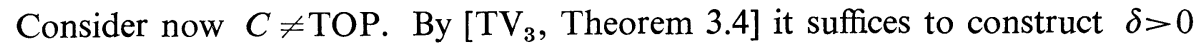
with the following weaker property (this reduction also holds for $C=$ TOP): If $f_{1}, f_{2}$ are as in the lemma, there is a C-homeomorphism $h: R^{n} \rightarrow R^{n}$ such that $d(h, \mathrm{id})<\varepsilon, h=\mathrm{id}$ on $Y=R^{n} \backslash N\left(f I^{n-1}(1 / 2), \varepsilon\right)$, and $h f_{1}=f_{2}$ on $I^{k} \times I^{m}(1 / 2)$. We apply the case $C=$ TOP of the lemma and 3.1. We first choose $\eta \in(0,1 / 2)$ and an open neighborhood $Y_{1}$ of $Y$ such that $\bar{Y}_{1} \cap f I^{n-1}(1 / 2+\eta)=\emptyset$. By the TOP case, there is $\delta>0$ with the following property: If $f_{1}, f_{2}: I^{n-1} \rightarrow R^{n}$ are locally flat embeddings such that $d\left(f_{i}, f\right)<\delta$ and $f_{1}=f_{2}$ on $\left(I^{k} \backslash I^{k}(1 / 2)\right) \times I^{m}$, there is a homeomorphism $g: R^{n} \rightarrow R^{n}$ such that $d(g, \mathrm{id})<\varepsilon / 2, g=\mathrm{id}$ near $\bar{Y}_{1}$, and $g f_{1}=f_{2}$ on $U=J^{k} \times J^{m}(1 / 2+\eta)$. We may assume that $\varepsilon$ and $\delta$ are so small that $f_{1}\left[I^{n-1} \backslash I^{n-1}(3 / 4)\right] \subset Y$.

We show that this $\delta$ is the desired number. Suppose that the last-mentioned $f_{1}, f_{2}$ are, in fact, locally $C$-flat. Choose an open set $V \subset R^{n}$ such that $f_{1} U$ is a closed subset of $V$; then $f_{2} U$ is closed in the open set $g V$. Define $\varepsilon_{1} \in C_{+}(V)$ by $2 \varepsilon_{1}(x)=\min \left(\varepsilon, d\left(g(x), R^{n} \backslash g V\right)\right)$. We apply 3.1 (c) with the substitution $C \mapsto C$, $Q_{1} \mapsto V, Q_{2} \mapsto g V, A \mapsto \bar{Y}_{1} \cap V, B, B^{\prime} \mapsto V, M_{i} \mapsto f_{i} U, f \mapsto g \mid V, \varepsilon \mapsto \varepsilon_{1}$. This is possible since $g$ extends the $C$-homeomorphism $f_{2} f_{1}^{-1}: f_{1} U \rightarrow f_{2} U$. We obtain a $C$-homeomorphism $g^{\prime}: V \rightarrow g V$ such that $g^{\prime} \in U\left(g \mid V, \varepsilon_{1}\right), g^{\prime}=g=\mathrm{id}$ on $Y_{1} \cap V$, and $g^{\prime}=g$ on $f_{1} U$. Extending $g^{\prime}$ by $g \mid R^{n} \backslash V$ we obtain a homeomorphism $g_{1}: R^{n} \rightarrow R^{n}$ such that 
$d\left(g_{1}, \mathrm{id}\right)<\varepsilon, g_{1} \mid Y_{1}=\mathrm{id}, g_{1} f_{1}=f_{2}$ on $U$, and $g_{1} \mid V$ is a $C$-embedding. By $\left[\mathrm{TV}_{3}\right.$, Theorem 4.4] there is a $C$-homeomorphism $h: R^{n} \rightarrow R^{n}$ such that $d(h, \mathrm{id})<\varepsilon$, $h \mid Y=\mathrm{id}$, and $h=g_{1}$ near $f_{1}\left[I^{k}(3 / 4) \times I^{m}(1 / 2)\right]$. Then $h f_{1}=f_{2}$ on $I^{k} \times I^{m}(1 / 2)$.

4.10. Lemma. Let $C, n, k, m, \varepsilon$ be as in 4.9 with $m \geqq 1$ and let $f: I_{+}^{n-1} \rightarrow R^{n}$ be an embedding. Then there is $\delta>0$ with the following property: If $f_{1}, f_{2}: I_{+}^{n-1} \rightarrow R^{n}$ are locally $C$-flat embeddings such that $d\left(f_{i}, f\right)<\delta$ and $f_{1}=f_{2}$ on $\left(I^{k} \backslash I^{k}(1 / 2)\right) \times I_{+}^{m}$, there is an ambient $\varepsilon$-isotopy $H$ of $R^{n}$ rel $R^{n} \backslash N\left(f I_{+}^{n-1}(1 / 2), \varepsilon\right)$ such that $H_{t}$ is a C-homeomorphism for each $t \in I$ and $H_{1} f_{1}=f_{2}$ on $I^{k} \times I_{+}^{m}(1 / 2)$.

Proof. We reduce the lemma to 4.9. We begin with some preliminary constructions. Let $N=N\left(f I_{+}^{n-1}(1 / 2), \varepsilon\right)$. Let $\sigma: R^{1} \rightarrow I^{1}$ and $\tau: R^{1} \rightarrow I$ be the unique nondecreasing retractions; then we define a retraction $r: R^{n} \rightarrow I_{+}^{n-1}$ by $r(x)=\left(\sigma\left(x_{1}\right), \ldots\right.$, $\left.\sigma\left(x_{n-2}\right), \tau\left(x_{n-1}\right)\right)$. For $s>0$, let $\quad B_{s}=I^{n-2}(1+s) \times[-s, 1+s] \times I^{1}(s) \subset R^{n} \quad$ and define homeomorphisms $\alpha_{s}, \beta_{s}: R^{1} \rightarrow R^{1}$ by

$$
\alpha_{s}(t)=\left\{\begin{array}{ll}
s t+s-1, & t \leqq-1 \\
t, & -1 \leqq t \leqq 1 \\
s t+1-s, & t \geqq 1
\end{array} \beta_{s}(t)= \begin{cases}s t, & t \leqq 0 \\
\alpha_{s}(t), & t \geqq 0 .\end{cases}\right.
$$

Then $\psi_{s}: R^{n} \rightarrow R^{n}, \psi_{s}(x)=\left(\alpha_{s}\left(x_{1}\right), \ldots, \alpha_{s}\left(x_{n-2}\right), \beta_{s}\left(x_{n-1}\right), s x_{n}\right)$, is a PL homeomorphism such that $\psi_{s} \mid I_{+}^{n-1}=\mathrm{id}, \psi_{s} B_{1}=B_{s}$, and $r \psi_{s}=r$. We choose $a \in(0,1)$ such that $|f r(x)-f r(y)|<\varepsilon / 15$ for all $x, y \in R^{n}$ with $|x-y|<a$. Consider $b \in(0, a / 2)$ and the subset

$$
A=I^{k} \times I^{m-1}(1 / 2+b) \times[b, 1 / 2] \cup\left(I^{k} \backslash J^{k}(1 / 2+b)\right) \times I^{m-1}(1 / 2+b) \times[0,1 / 2]
$$

of $I_{+}^{n-1}$. It is obvious that if $b$ is sufficiently small, then $f I_{+}^{n-1}(1 / 2+b) \subset N$ and there is an ambient $a$-isotopy $\Gamma$ of $R^{n}$ rel $R^{n} \backslash N_{0}$, where $N_{0}=N\left(I^{n-2}(1 / 2), a\right)$, such that $\Gamma_{t}$ is PL and $\Gamma_{t} I_{+}^{n-1} \subset I_{+}^{n-1}$ for each $t \in I$ and that $\Gamma_{1}\left[I^{k} \times I_{+}^{m}(1 / 2)\right] \subset A$. We fix such a number $b$.

Obviously, there is a PL homeomorphism $\varphi: I^{n-1} \rightarrow I_{\perp+}^{n-1}$ such that

$$
\begin{gathered}
\varphi\left[\left(I^{k} \backslash I^{k}(1 / 2)\right) \times I^{m}\right]=\left(I^{k} \backslash I^{k}(1 / 2)\right) \times I_{+}^{m}, \\
\varphi I^{n-1}(1 / 2)=I^{k}(1 / 2) \times I^{m-1}(1 / 2+b) \times[b, 1 / 2] \subset I_{+}^{n-1}(1 / 2+b), \\
\varphi\left[I^{k} \times I^{m}(1 / 2)\right]=A .
\end{gathered}
$$

Thus by 4.9 there is $\delta_{0}>0$ with the following property: If $f_{1}, f_{2}: I_{+}^{n-1} \rightarrow R^{n}$ are locally $C$-flat embeddings such that $d\left(f_{i}, f\right)<\delta_{0}$ and $f_{1}=f_{2}$ on $\left(I^{k} \backslash I^{k}(1 / 2)\right) \times I_{+}^{m}$, there is an ambient ( $(\varepsilon / 3)$-isotopy $H^{0}$ of $R^{n}$ rel $R^{n} \backslash N$ such that $H_{t}^{0}$ is a $C$-homeomorphism for each $t \in I$ and that $H_{1}^{0} f_{1}=f_{2}$ on $A$, whence $H_{1}^{0} f_{1} \Gamma_{1}=f_{2} \Gamma_{1}$ on $I^{k} \times I_{+}^{m}(1 / 2)$.

We show that for every locally $C$-flat embedding $g: I_{+}^{n-1} \rightarrow R^{n}$ with $d(g, f)<$ $\varepsilon / 15$ there is an ambient ( $(\varepsilon / 3)$-isotopy $G$ of $R^{n}$ rel $R^{n} \backslash N$ such that $G_{t}$ is a $C$ homeomorphism for each $t \in I$ and $G_{1} g=g \Gamma_{1} \mid I_{+}^{n-1}$. By [Ru, Corollary 3.4.1] 
if $C=\mathrm{TOP}$ or by [GV, Theorem 4.3] if $C \neq \mathrm{TOP}$, there is a $C$-homeomorphism $g^{\prime}: R^{n} \rightarrow R^{n}$ extending $g$. Since $d\left(r \mid B_{s}\right.$, id $) \rightarrow 0$ as $s \rightarrow 0$, there is $s>0$ such that $d\left(g^{\prime}\left|B_{s}, g r\right| B_{s}\right)<\varepsilon / 15$. Then $\bar{g}=g^{\prime} \psi_{s}: R^{n} \rightarrow R^{n}$ is a $C$-homeomorphism extending $g$ with $d\left(\bar{g}\left|B_{1}, g r\right| B_{1}\right)=d\left(g^{\prime} \psi_{s}\left|B_{1}, g r \psi_{s}\right| B_{1}\right)=d\left(g^{\prime}\left|B_{s}, g r\right| B_{s}\right)<\varepsilon / 15$. We set $G_{t}=$ $\bar{g} \Gamma_{t} \bar{g}^{-1}$ for $t \in I$. Then $G_{t} \mid R^{n} \backslash \bar{g} N_{0}=$ id and $G_{t} g=g \Gamma_{t} \mid I_{+}^{n-1}$. If $x \in R^{n}, y \in I^{n-2}(1 / 2)$, and $|x-y|<a$,

$$
\begin{aligned}
|\bar{g}(x)-f(y)| & \leqq|\bar{g}(x)-g r(x)|+|g r(x)-f r(x)|+|f r(x)-f r(y)| \\
& <3 \cdot \varepsilon / 15=\varepsilon / 5,
\end{aligned}
$$

whence $\bar{g} N_{0} \subset N$. If $x \in N_{0} \subset B_{1}$,

$$
\begin{aligned}
\left|\bar{g} \Gamma_{t}(x)-\bar{g}(x)\right| & \leqq 2 d\left(\bar{g}\left|B_{1}, g r\right| B_{1}\right)+2 d(g, f)+\left|f r \Gamma_{t}(x)-f r(x)\right| \\
& <5 \cdot \varepsilon / 15=\varepsilon / 3 .
\end{aligned}
$$

Hence $G$ is the desired isotopy.

We show that $\delta=\min \left(\delta_{0}, \varepsilon / 15\right)$ satisfies the lemma. Let $f_{1}, f_{2}$ be as in the lemma. By what we have proved, there are ambient ( $\varepsilon / 3$ )-isotopies $H^{0}, H^{1}, H^{2}$ of $R^{n}$ rel $R^{n} \backslash N$ such that $H_{t}^{i}$ is a $C$-homeomorphism for all $t \in I$ and $i=0,1,2$, that $H_{1}^{0} f_{1} \Gamma_{1}=f_{2} \Gamma_{1}$ on $I^{k} \times I_{+}^{m}(1 / 2)$, and that $H_{1}^{i} f_{i}=f_{i} \Gamma_{1} \mid I_{+}^{n-1}, i=1,2$. Then $H=\left(H^{2}\right)^{-1} * H^{0} * H^{1}$ is the desired isotopy.

The rest of the proof of 4.1 will now be standard. The next lemma is the second stage of this proof.

4.11. Lemma. Let $C \in\{$ TOP, LQC, LIP $\}$ and $n \neq 4$, let $G$ be an open subset of $R_{+}^{n-1}$, let $A, B, U$ be open in $G$ such that $\bar{A} \cap G \subset U, \bar{B} \subset G$, and $\bar{B}$ is compact, let $f: G \rightarrow R^{n}$ be an embedding, and let $\varepsilon>0$. Then there is $\delta>O$ with the following property: If $f_{1}, f_{2}: G \rightarrow R^{n}$ are locally $C$-flat embeddings such that $d\left(f_{i}, f\right)<\delta$ and $f_{1}\left|U=f_{2}\right| U$, there is an ambient $\varepsilon$-isotopy $\varphi$ of $R^{n}$ rel $R^{n} \backslash N(f B, \varepsilon)$ such that $\varphi_{t}$ is a C-homeomorphism for each $t \in I$ and $\varphi_{1} f_{1}=f_{2}$ on $A \cup B$.

Proof. Let $A_{1}=\bar{A} \cap G$ and $N=N(f B, \varepsilon)$. Choose a compact PL $(n-1)$ manifold $P \subset f^{-1} N$ with $\bar{B} \subset \operatorname{int}_{G} P$. Choose a triangulation $\left(K, K_{0}\right)$ of $(P, \partial P)$. Let $K^{\prime}$ be the first barycentric subdivision of $K$ and $K^{\prime \prime}$ the second one. For $\sigma \in K$, let $\hat{\sigma}$ be the barycenter of $\sigma, H_{\sigma}=\cup\left\{\tau \mid \hat{\sigma} \in \tau \in K^{\prime \prime}\right\}$, and $i\left(H_{\sigma}\right)=\operatorname{dim} \sigma$. Write $K=\left\{\sigma_{1}, \ldots, \sigma_{r}\right\} \quad$ with $\operatorname{dim} \sigma_{j} \leqq \operatorname{dim} \sigma_{j+1}$. Then $\quad \mathscr{H}=\left\{H_{\sigma_{1}}, \ldots, H_{\sigma_{r}}\right\} \quad$ is a handle decomposition of $P$ as in [H, pp. 224-227], [RS, p. 82] or [Ru, pp. 31-33]. In fact, if $\sigma \in K, P_{\sigma}=\cup\left\{H_{\tau} \mid \tau \in K, \operatorname{dim} \tau<\operatorname{dim} \sigma\right\}, \tilde{H}_{\sigma}=\operatorname{cl}\left(\left(\cup\left\{\tau \mid \hat{\sigma} \in \tau \in K^{\prime}\right\}\right) \backslash\right.$ $\left.P_{\sigma}\right), k=i\left(H_{\sigma}\right)$, and $m=n-1-k$ (thus $m \geqq 1$ whenever $\left.\sigma \in K_{0}\right)$, it is easy to show that $\left(\tilde{H}_{\sigma}, H_{\sigma}, \tilde{H}_{\sigma} \cap P_{\sigma}, \tilde{H}_{\sigma} \cap \partial P\right)$ is PL homeomorphic either to $\left(I^{k} \times 2 I^{m}, I^{k} \times I^{m}\right.$, $\left.\partial I^{k} \times 2 I^{m}, \emptyset\right)$ whenever $\sigma \in K \backslash K_{0} \quad$ or to $\left(I^{k} \times 2 I_{+}^{m}, I^{k} \times I_{+}^{m}, \partial I^{k} \times 2 I_{+}^{m}, I^{k} \times 2 I^{m-1}\right)$ whenever $\sigma \in K_{0}$. Setting $H_{\sigma} \leqq H_{\tau}$ if $H_{\sigma} \cap H_{\tau} \neq \emptyset$ and $i\left(H_{\sigma}\right) \leqq i\left(H_{\tau}\right)$ (i.e., if $\sigma$ is a face of $\tau$ ) we obtain a partial order for $\mathscr{H}$. Let $\mathscr{H}_{0}$ and $\mathscr{H}_{1}$, respectively, be the sets of the handles $H \in \mathscr{H}$ such that there is $H^{\prime} \in \mathscr{H}$ with $H \leqq H^{\prime}$ and with either 
$H^{\prime} \cap A_{1} \neq \emptyset$ or $H^{\prime} \cap \bar{B} \neq \emptyset$, respectively. Then $H \leqq H^{\prime} \in \mathscr{H}_{i}$ implies $H \in \mathscr{H}_{i}$ for $i=0,1$. We may assume that $\cup \mathscr{H}_{0} \subset U$ and $\cup \mathscr{H}_{1} \subset$ int $_{G} P$. Observe that $\left(\right.$ int $\left._{G} P\right) \cap \partial P=\left(\right.$ int $\left._{G} P\right) \cap R^{n-2}$. Write $\mathscr{H}_{1} \backslash \mathscr{H}_{0}=\left\{H_{1}, \ldots, H_{s}\right\}$ with $i\left(H_{j}\right) \leqq i\left(H_{j+1}\right)$. Let $\mathscr{H}_{j}^{\prime}=\left(\mathscr{H}_{0} \cap \mathscr{H}_{1}\right) \cup\left\{H_{1}, \ldots, H_{j}\right\}$ and $P_{j}=\cup \mathscr{H}_{j}^{\prime}$ for $0 \leqq j \leqq s$. Then $P_{s} \supset B$. For each $H \in \mathscr{H}$ with $H \cap H_{j} \neq \emptyset$ we have $i(H)<i\left(H_{j}\right)$ if and only if $H \in \mathscr{H}_{j-1}^{\prime}$.

We set $U_{0}=U \supset A_{1} \cup P_{0}$. Inductively, for each $j \in\{1, \ldots, s\}$ we first choose either a PL embedding $\mu_{j}: I^{n-1} \rightarrow f^{-1} N \backslash A_{1}$ whenever $H_{j} \cap R^{n-2}=\emptyset$ or a PL embedding $\mu_{j}: I_{+}^{n-1} \rightarrow f^{-1} N \backslash A_{1}$ whenever $H_{j} \cap R^{n-2} \neq \emptyset$ and then define an open neighborhood $U_{j}$ of $A_{1} \cup P_{j}$ in $G$ such that, setting $k=i\left(H_{j}\right), m=n-1-k$, we have either

$$
\begin{gathered}
\mu_{j}^{-1} R^{n-2}=\emptyset, \\
\mu_{j}\left[I^{k} \times I^{m}(1 / 3)\right]=H_{j}, \\
\mu_{j} I^{n-1} \cap P_{j-1}=\mu_{j}\left[\partial I^{k} \times I^{m}\right], \\
\mu_{j}\left[\left(I^{k} \backslash I^{k}(1 / 2)\right) \times I^{m}\right] \subset U_{j-1}, \\
U_{j}=\left(U_{j-1} \backslash \mu_{j}\left[I^{k}(2 / 3) \times I^{m}\right]\right) \cup \mu_{j}\left[I^{k} \times J^{m}(1 / 2)\right]
\end{gathered}
$$

whenever $H_{j} \cap R^{n-2}=\emptyset$ or

$$
\begin{gathered}
\mu_{j}^{-1} R^{n-2}=I^{n-2}, \\
\mu_{j}\left[I^{k} \times I_{+}^{m}(1 / 3)\right]=H_{j}, \\
\mu_{j} I_{+}^{n-1} \cap P_{j-1}=\mu_{j}\left[\partial I^{k} \times I_{+}^{m}\right], \\
\mu_{j}\left[\left(I^{k} \backslash I^{k}(1 / 2)\right) \times I_{+}^{m}\right] \subset U_{j-1}, \\
U_{j}=\left(U_{j-1} \backslash \mu_{j}\left[I^{k}(2 / 3) \times I_{+}^{m}\right]\right) \cup \mu_{j}\left[I^{k} \times J_{+}^{m}(1 / 2)\right]
\end{gathered}
$$

whenever $H_{j} \cap R^{n-2} \neq \emptyset$. In both cases, the image of $\mu_{j}$ is locally PL flat in $G$. Choose numbers $0<\delta_{j} \leqq \varepsilon_{j} \leqq \varepsilon / s$ for $1 \leqq j \leqq s$ such that $\varepsilon_{j} \leqq \delta_{j+1} / 2$ for $j<s$, that either

$$
N_{j}=N\left(f \mu_{j} I^{n-1}(1 / 2), \varepsilon_{j}\right) \subset N \backslash N\left(f\left[G \backslash \mu_{j}\left[I^{k}(2 / 3) \times I^{m}\right]\right], \delta_{j}\right)
$$

if $H_{j} \cap R^{n-2}=\emptyset$ or

$$
N_{j}=N\left(f \mu_{j} I_{+}^{n-1}(1 / 2), \varepsilon_{j}\right) \subset N \backslash N\left(f\left[G \backslash \mu_{j}\left[I^{k}(2 / 3) \times I_{+}^{m}\right]\right], \delta_{j}\right)
$$

if $H_{j} \cap R^{n-2} \neq \emptyset$, where $k, m$ are as above, and that $\delta_{j}$ is the number given either by 4.9 if $H_{j} \cap R^{n-2}=\emptyset$ or by 4.10 if $H_{j} \cap R^{n-2} \neq \emptyset$ with the substitution $(C, k, m) \mapsto$ $(C, k, m), f \mapsto f \mu_{j}, \varepsilon \mapsto \varepsilon_{j}$. Then $\delta=\delta_{1}$ satisfies the lemma. In fact, let $f_{1}, f_{2}$ be as in the lemma. Then it follows inductively that there exist ambient $\varepsilon_{j}$-isotopies $\varphi^{j}, 1 \leqq j \leqq s$, of $R^{n}$ rel $R^{n} \backslash N_{j}$ such that $\varphi_{t}^{j}$ is a $C$-homeomorphism for all $t$ and $\varphi_{1}^{j} \ldots \varphi_{1}^{1} f_{1}=f_{2}$ on $U_{j}$. Hence $\varphi=\varphi^{s} * \ldots * \varphi^{1}$ is the desired isotopy.

4.12. Proof of 4.1 for $n \geqq 2$. We may assume that $B$ is closed and $U \subset B$. Choose an open set $Q_{0} \subset Q$ such that $f$ defines a closed embedding $f^{\prime}: B \rightarrow Q_{0}$, $x \mapsto f(x)$. We may assume that $N \subset \operatorname{int} Q_{0}$ and that $N$ is closed either in $Q_{0}$ if 
$C=$ TOP or in $Q$ if $C \neq$ TOP. Then there is $\varepsilon^{\prime} \in C_{+}\left(Q_{0}\right)$ such that $\varepsilon^{\prime} \mid N \leqq \varepsilon$ and $\varepsilon^{\prime}(x) \leqq d\left(x, Q \backslash Q_{0}\right) / 2$ for each $x \in Q_{0}$. There is a locally finite family $\mathscr{V}=\mathscr{V}_{0} \cup \mathscr{V}^{\prime}$ of open subsets of $M$ in $M$, where $\mathscr{V}_{0}=\{U\}$, such that $A \subset \cup \mathscr{V}$ and that for each $V \in \mathscr{V}^{\prime}, V$ is $C$-homeomorphic to an open set in $R_{+}^{n-1}, \bar{V}$ is compact in $B$, and $f \bar{V}$ is contained in an open subset $Q_{V} \subset N$ of $Q C$-homeomorphic to $R^{n}$. By [Mu, Lemma 2.7] we may assume that $\mathscr{V}^{\prime}$ has a partition $\mathscr{V}^{\prime}=\mathscr{V}_{1} \cup \ldots \cup \mathscr{V}_{n}$ with each family $\mathscr{V}_{j}$ being disjoint. We choose for each $V \in \mathscr{V}$ open sets $V=V^{-1} \supset V^{0} \supset \ldots \supset V^{n}$ such that $\bar{V}^{j} \subset V^{j-1}$ and that $A \subset \cup\left\{V^{n} \mid V \in \mathscr{V}\right\}$. Define open sets

$$
\begin{aligned}
& D_{j}=\cup\left\{V^{j-i} \mid 0 \leqq i \leqq j, V \in \mathscr{V}_{i}\right\} \subset B \quad(0 \leqq j \leqq n), \\
& D_{j}^{\prime}=\cup\left\{V^{j-i+1} \mid 0 \leqq i \leqq j, V \in \mathscr{V}_{i}\right\} \subset B \quad(0 \leqq j<n)
\end{aligned}
$$

in $M$. Then $D_{0}=U^{0}, D_{n} \supset A$, and for each $1 \leqq j \leqq n$ we have $\bar{D}_{j-1}^{\prime} \subset D_{j-1}$ and

$$
D_{j}=D_{j-1}^{\prime} \cup\left(\cup\left\{V^{0} \mid V \in \mathscr{V}_{j}\right\}\right) .
$$

Consider $j \in\{1, \ldots, n\}$ and a function $\varepsilon_{j} \in C_{+}\left(Q_{0}\right)$, to be specified below. For each $V \in \mathscr{V}_{j}$ we choose $\varepsilon_{V}>0$ such that $N_{V}=\operatorname{cl} N\left(f V^{0}, \varepsilon_{V}\right)$ is compact in $Q_{V}$, $\varepsilon_{V} \leqq \min \varepsilon_{j} N_{V}, N_{V} \cap f[B \backslash V]=\emptyset$, and that $\left\{N_{V} \mid V \in \mathscr{V}_{j}\right\}$ is a locally finite disjoint family in $Q_{0}$. It is easy to see that 4.11 also holds if the embedding $f: G \rightarrow R^{n}$ is replaced by the embedding $f \mid V: V \rightarrow Q_{V}$ for $V \in \mathscr{V}_{j}$. Let $\delta_{V}>0$ with $\delta_{V} \leqq$ $d\left(f V, Q \backslash Q_{V}\right)$ be the number given by this version of 4.11 with the substitution $A \mapsto D_{j-1}^{\prime} \cap V, B \mapsto V^{0}, U \mapsto D_{j-1} \cap V, \varepsilon \mapsto \varepsilon_{V}$. There is $\delta_{j} \in C_{+}(B)$ such that $\max \delta_{j} \bar{V} \leqq \delta_{V}$ and $\delta_{j}(x) \leqq d\left(f(x), N_{V}\right)$ for all $V \in \mathscr{V}_{j}$ and $x \in B \backslash V$. We fix the functions $\varepsilon_{1}, \delta_{1}, \ldots$, $\varepsilon_{n}, \delta_{n}$ so small that

$$
\begin{gathered}
U\left(\mathrm{id}_{Q_{0}}, \varepsilon_{n}\right) \circ \ldots \circ U\left(\mathrm{id}_{Q_{0}}, \varepsilon_{1}\right) \subset U\left(\mathrm{id}_{Q_{0}}, \varepsilon^{\prime}\right), \\
U\left(\mathrm{id}_{Q_{0}}, \varepsilon_{j}\right) \circ U\left(f^{\prime}, \delta_{j}\right) \subset U\left(f^{\prime}, \delta_{j+1}\right) \text { if } 1 \leqq j<n .
\end{gathered}
$$

There is $\delta \in C_{+}(M)$ such that $\delta(x) \leqq \min \left(\delta_{1}(x), d\left(f(x), Q \backslash Q_{0}\right)\right)$ for each $x \in B$ and $N(A, \delta) \subset D_{n}$.

We show that this $\delta$ satisfies the theorem. Let $f_{1}, f_{2}$ be as in 4.1. Then $f_{i}$ defines an embedding $f_{i}^{\prime}: B \rightarrow Q_{0}, x \mapsto f_{i}(x)$. We construct inductively ambient $\varepsilon_{j}$-isotopies $H^{j}, 1 \leqq j \leqq n$, of $Q_{0}$ rel $Q_{0} \backslash N$ such that $H_{t}^{j}$ is a $C$-homeomorphism for all $t$ and $j$ and that $H_{1}^{j} \ldots H_{1}^{1} f_{1}^{\prime}=f_{2}^{\prime}$ on $D_{j}$ for each $j$. The desired isotopy $H$ is then the union of $H^{n} * \ldots * H^{1}$ and of the trivial ambient isotopy of $Q \backslash Q_{0}$. Let $1 \leqq j \leqq n$. The embedding $g_{j-1}=H_{1}^{j-1} \ldots H_{1}^{1} f_{1}^{\prime}: B \rightarrow Q_{0} \quad\left(g_{0}=f_{1}^{\prime}\right)$ is locally $C$-flat on $\operatorname{int}_{M} B, g_{j-1}, f_{2}^{\prime} \in U\left(f^{\prime}, \delta_{j}\right)$, and $g_{j-1}=f_{2}^{\prime}$ on $D_{j-1}$. Then for each $V \in \mathscr{V}_{j}$ there is, by the definition of $\delta_{V}$, an ambient $\varepsilon_{V}$-isotopy $H^{V}$ of $Q_{0}$ rel $Q_{0} \backslash N_{V}$ such that $H_{t}^{V}$ is a $C$-homeomorphism for each $t$ and $H_{1}^{V} g_{j-1}=f_{2}^{\prime}$ on $D_{j} \cap V$. We set $H_{t}^{j}(x)=H_{t}^{V}(x)$ for each $t \in I$ whenever there is $V \in \mathscr{V}_{j}$ with $x \in N_{V}$ and $H_{t}^{j}(x)=x$ otherwise. Then $H^{j}$ is an ambient $\varepsilon_{j}$-isotopy of $Q_{0}$ rel $Q_{0} \backslash N$ such that $H_{t}^{j}$ is a $C$-homeomorphism for each $t$. We finally show that if $x \in D_{j}$, then $H_{1}^{j} g_{j-1}(x)=f_{2}^{\prime}(x)$. If $x \in V \in \mathscr{V}_{j}$, then for each $W \in \mathscr{V}_{j}, W \neq V$, we have $x \notin W$, 
whence $\quad d\left(g_{j-1}(x), f(x)\right)<\delta_{j}(x) \leqq d\left(f(x), N_{W}\right)$, and thus $g_{j-1}(x) \notin N_{W}$. Hence $H_{1}^{j} g_{j-1}(x)=H_{1}^{V} g_{j-1}(x)=f_{2}^{\prime}(x)$. If $x \notin \cup \mathscr{V}_{j}$, then $g_{j-1}(x) \notin \cup\left\{N_{V} \mid V \in \mathscr{V}_{j}\right\}$ and $x \in D_{j-1}^{\prime}$, whence $H_{1}^{j} g_{j-1}(x)=g_{j-1}(x)=f_{2}^{\prime}(x)$.

4.13. Lemma. Let $Q=R_{+}^{1} \times R^{1}$, let $f:[0,2] \rightarrow Q$ be an embedding with $f^{-1} \partial Q=\{0\}$, and let $\varepsilon>0$. Then there is $\delta>0$ with the following property: If $f_{1}, f_{2}:[0,2] \rightarrow Q$ are embeddings such that $f_{i}^{-1} \partial Q=\{0\}$ and $d\left(f_{i}, f\right)<\delta$, there is an $\varepsilon$-push $h$ of $(Q, f I)$ with $h f_{1}=f_{2}$ on $I$.

Proof. Since $f$ can be extended to a selfhomeomorphism of $Q$, it suffices to prove the modification of 4.7 , with the additional assertion omitted, where $\left(R^{2},[-1,2]\right)$ is replaced by $(Q,[0,2])$ and where $f_{i}^{-1} \partial Q=\{0\}$ (it is not difficult to construct the isotopy). The proof is otherwise the same as earlier, but we now define $A_{i 0 k}$ to be the segment in $\partial Q$ whose endpoints are $f_{i}(0)$ and $\left(0,(-1)^{k} \eta\right)$.

4.14. Lemma. The modification of 4.10 is true where $n=2$ or $3, R^{n}$ is replaced by $R_{+}^{n}, f_{i}^{-1} R^{n-1}=f^{-1} R^{n-1}=I^{n-2}$, and, whenever $C \neq \mathrm{TOP}, H_{t}$ is not claimed to be a C-homeomorphism for $t \neq 1$.

Proof. For $C=$ TOP, $n=2$, this is proved in 4.13. The case $C=$ TOP, $n=3$ follows from 4.5. For $C \neq \mathrm{TOP}$, the lemma can be proved by the aid of the TOP case, 3.1(b), and $\left[\mathrm{TV}_{3}\right.$, Theorem 4.8] as the case $C \neq \mathrm{TOP}$ of 4.9 (construct the isotopy gradually).

4.15. Lemma. The modification of 4.11 is true where $n=2$ or $3, R^{n}$ is replaced by $R_{+}^{n}, f_{i}^{-1} R^{n-1}=f^{-1} R^{n-1}=G \cap R^{n-2}$, and, whenever $C \neq \mathrm{TOP}, \varphi_{i}$ is not claimed to be a C-homeomorphism for $t \neq 1$.

Proof. The proof is a slight modification of that of 4.11 ; Lemmas 4.9 and 4.14 are applied.

4.16. Lemma. The modification of 4.9 is true where $C=\mathrm{TOP}, n=3, k=0$, $\left(R^{n}, I^{n-1}, I^{n-1}(1 / 2)\right)$ is replaced by $\left(R_{+}^{3}, I \times I,[0,1 / 2] \times[0,1 / 2]\right)$, and $f_{i}^{-1} R^{2}=$ $f^{-1} R^{2}=I$.

Proof. This follows from 4.5.

4.17. Lemma. The modification of 4.11 is true where $C=\mathrm{TOP}, n=3,\left(R^{n}, R_{+}^{n-1}\right)$ is replaced by $\left(R_{+}^{3}, R_{+}^{1} \times R_{+}^{1}\right)$, and $f_{i}^{-1} R^{2}=f^{-1} R^{2}=G \cap R_{+}^{1}$.

Proof. The proof is a slight modification of that of 4.11; Lemmas 4.9, 4.10, 4.14 , and 4.16 are applied.

4.18. Proof of 4.2 for $n \geqq 2$. The proof is an obvious modification of 4.12; Lemmas 4.11, 4.15, and, whenever $C=\mathrm{TOP}, n=3$, also 4.17 are applied. 


\section{Locally $C$-flat approximation}

In this section we first prove the following three theorems. The first two of them deal with the relative locally $C$-flat approximation of embeddings or of immersions, respectively, and the third one with the extension of locally $C$-flat approximations. We then give two applications, 5.10 and 5.11, on "LQC or LIP taming" of locally flat embeddings. For a discussion on 5.1-5.3, see 5.4.

5.1. Theorem. Let $C \in\{$ TOP, LQC, LIP $\}$ and $n \neq 4$, let $M$ be an $(n-1)$ and $Q$ an n-dimensional $C$-manifold with $\partial Q=\emptyset$, let $A \subset M$ be closed, let $f: M \rightarrow Q$ be an embedding which is locally $C$-flat near $A$, and let $\varepsilon \in C_{+}(M)$. Then there is a locally $C$-flat embedding $f^{*}: M \rightarrow Q$ in $U(f, \varepsilon)$ such that $f^{*}=f$ near $A$.

5.2. Theorem. Let $C, n, M, Q, A$ be as in 5.1, let $B \subset M$ be closed and $B^{\prime} \subset M$ open with $B \subset B^{\prime}$, let $f: M \rightarrow Q$ be an immersion which is locally $C$-flat near $A$, and let $\varepsilon \in C_{+}\left(B^{\prime}\right)$. Then there is an immersion $f^{*}: M \rightarrow Q$ such that $f^{*}=f$ near $A$ and on $M \backslash B^{\prime}$, that $f^{*} \mid B^{\prime} \in U\left(f \mid B^{\prime}, \varepsilon\right)$, and that $f^{*}$ is locally $C$-flat near $A \cup B$.

5.3. Theorem. Let $C, n, M, Q, A, \varepsilon$ be as in 5.1, let $U \subset M$ be an open neighborhood of $A$, and let $f: M \rightarrow Q$ be an embedding. Then there is $\delta \in C_{+}(M)$ with the following property: If $f_{0}: U \rightarrow Q$ is a locally $C$-flat embedding in $U(f|U, \delta| U)$, there is a locally $C$-flat embedding $f^{*}: M \rightarrow Q$ in $U(f, \varepsilon)$ such that $f^{*}=f_{0}$ near $A$.

5.4. Remarks. 1. Theorem 5.1 implies immediately the following version of 5.2, which generalizes 5.1: Suppose that $f$ in 5.2 is an embedding (and $B=\emptyset$ ); then $f^{*}$ can be chosen to be an embedding such that $f^{*} \mid B^{\prime}$ is locally $C$-flat. The sets $B$ and $B^{\prime}$ of 5.1 and 5.2 could similarly be included in the analogous result [LT, Theorem 4.4]; see 5.9.

2. Several special cases of 5.1 and 5.2 are known. In particular, for $C=$ TOP, $n \geqq 5$, and $A=\emptyset$, Theorem 5.1 has been proved by Ancel and Cannon [AC, p. 61]. (In $[\mathrm{AC}]$ this result is only stated in the case $\partial M=\emptyset$, but its general case $[\mathrm{D}$, Theorem 5B.1] is obtained as follows. Using a collar of $\partial M$ in $M$ construct a closed locally flat embedding $h: M \rightarrow$ int $M$ sufficiently close to id. Choose a locally flat embedding $g$ : int $M \rightarrow Q$ sufficiently close to $f \mid$ int $M$. Then $f^{*}=g h$ is the desired embedding.) Theorems 5.1 and 5.2 hold with $f^{*}=f$ in the case $n=1$ trivially and in the case $C=\mathrm{TOP}, n=2$ by the Schoenflies theorem. For $C \in\{\mathrm{LQS}, \mathrm{LIP}\}$ and $n=2$, 5.1 and 5.2 follow from the relative $C$-approximation result [LT, Theorem 4.4] (cf. 5.4.1) and the $C$-flatness of $C$-embeddings $I^{1} \rightarrow R^{2}$ ([Ri, Theorem 1], [ $\mathrm{T}_{2}$, Theorem $\mathrm{B}]$ ); however, we will give a new proof for this special case. Theorem 5.1 for $C=$ TOP, $n=3$ follows easily from Bing's results [ $\mathrm{B}_{1}$, Theorem 9] and [ $\mathrm{B}_{2}$, Theorems 7 and 8]. In the case $C \in\{\mathrm{LQC}, \mathrm{LIP}\}, n=3$, and $A=\emptyset$, we may assume that $M$ and $Q$ are PL manifolds; hence 5.1 now follows from the fact that $f$ can even be 
approximated by PL embeddings, by the case $C=\mathrm{TOP}$ and $\left[\mathrm{B}_{1}\right.$, Theorem 6 and its proof].

3. To prove 5.1 and 5.3 for $C=\mathrm{TOP}, n \geqq 5$, we apply, together with 4.1 , the Ancel-Cannon theorem in its general form, mentioned above. However, a slight reorganization of this section shows that the special case $(M, Q)=\left(R^{n-1}, R^{n}\right)$ of this result would suffice. In fact, the special case $(M, Q)=\left(I^{n-1}, R^{n}\right)$ is enough in a more laborious proof.

\subsection{Lemma. Theorem 5.3 holds if $C=$ TOP.}

Proof. We may assume that $f$ is closed. Then there are $\eta \in C_{+}(Q)$ and $\delta_{0} \in C_{+}(M)$ with $U\left(\mathrm{id}_{Q}, \eta\right) \circ U\left(f, \delta_{0}\right) \subset U(f, \varepsilon)$. Choose a closed neighborhood $B$ of $A$ with $B \subset U$. Let $\delta_{1} \in C_{+}(U)$ be the function given by 4.1 with the substitution $C \mapsto \mathrm{TOP}, n \mapsto n, M \mapsto U, Q \mapsto Q, A \mapsto A, B \mapsto B, U \mapsto \emptyset, f \mapsto f \mid U, N \mapsto Q, \varepsilon \mapsto \eta$. There is $\delta \in C_{+}(M)$ such that $\delta \leqq \delta_{0}$ and $\delta\left|B \leqq \delta_{1}\right| B$. We show that this $\delta$ satisfies 5.3. Let $f_{0}$ be as in 5.3. By the special case $C=\mathrm{TOP}, A=\emptyset$ of 5.1, which is known to be true (see 5.4.2), there is a locally flat embedding $f_{1}: M \rightarrow Q$ in $U(f, \delta)$. Then by 4.1 there is a homeomorphism $h: Q \rightarrow Q$ in $U(\mathrm{id}, \eta)$ such that $h f_{1}=f_{0}$ near $A$. Hence $f^{*}=h f_{1}$ is the desired embedding.

\subsection{Lemma. Theorem 5.1 holds if $C=\mathrm{TOP}$.}

Proof. This follows from 5.5.

5.7. Lemma. For $C \neq \mathrm{TOP}, 5.1$ holds if $M=R^{n-1}$ or $R_{+}^{n-1}$ and if there is an embedding $F: R^{n} \rightarrow Q$ extending $f$.

Proof. Choose an open neighborhood $U$ of $A$ in $R^{n}$ such that $f \mid U \cap M$ is locally $C$-flat. By 3.1 if $M=R^{n-1}$ or by 3.3 if $M=R_{+}^{n-1}$, there is a $C$-embedding $F_{1}: U \rightarrow Q$ extending $f \mid U \cap M$ such that $F_{1}$ can be extended by $F \mid R^{n} \backslash U$ to an embedding $F_{2}: R^{n} \rightarrow Q$. Then $F_{2} \mid M=f$. By $\left[\mathrm{TV}_{3}\right.$, Theorem 4.4] there is a $C$-embedding $F^{*}: R^{n} \rightarrow Q$ such that $F^{*} \mid M \in U(f, \varepsilon)$ and $F^{*}=F_{2}$ near $A$. Then $f^{*}=F^{*} \mid M$ is the desired locally $C$-flat embedding.

5.8. Lemma. Let $C \in\{$ TOP, LQC, LIP $\}$ and $n \neq 4$, let $(X, Y)$ be either $\left(2 J^{n-1}, J^{n-1}\right)$ or $\left(2 J_{+}^{n-1}, J_{+}^{n-1}\right)$, let $f: X \rightarrow R^{n}$ be an embedding which is locally $C$-flat near a closed subset $A$ of $X$, let $B \subset Y$ be compact, and let $\varepsilon>0$. Then there is an embedding $f^{*}: X \rightarrow R^{n}$ such that $f^{*}=f$ on $X \backslash Y$ and near $A, d\left(f^{*}, f\right)<\varepsilon$, and $f^{*}$ is locally $C$-flat near $A \cup B$.

Proof. Choose a closed neighborhood $A_{1}$ of $A$ in $X$ such that $f$ is locally $C$-flat near $A_{1}$. By 5.6 there is a locally flat embedding $g: Y \rightarrow R^{n}$ such that $g=f$ on $A_{1} \cap Y$ and that $g$ is so close to $f \mid Y$ that $f_{1}=g \cup(f \mid X \backslash Y)$ is an embedding with $d\left(f_{1}, f\right)<\varepsilon$. Then $f_{1}=f$ on $A_{1}$. Hence $f^{*}=f_{1}$ satisfies the lemma for $C=$ TOP. 
Consider $C \in\{$ LQC, LIP $\}$. We may replace $f$ by $f_{1}$ and, hence, assume that $f \mid Y$ is locally flat. Choose $r \in(0,1)$ with $B \subset r Y$. Then $f \mid r \bar{Y}$ is locally flat. We may well replace $Y$ by $r Y$ in the lemma. Thus we may assume that $f \mid \bar{Y}$ is locally flat. Then by [Ru, Corollary 3.4.1] there is a homeomorphism $F: R^{n} \rightarrow R^{n}$ extending $f \mid \bar{Y}$. Thus, if we repeat the above construction of $f_{1}$, we may assume by 5.7 that $g$ is locally $C$-flat. Then again $f^{*}=f_{1}$ satisfies the lemma.

5.9. Proof of 5.1, 5.2, and 5.3. For $C=$ TOP, the proof is only needed for 5.2 by 5.5 and 5.6. The joint proof of 5.1 and of the special case $B=M$ of 5.2 is almost the same as that of [LT, Theorem 4.4]; it is only based on 5.8 in place of the analogous [LT, Lemma 4.2]. To obtain the general case of 5.2, we modify this proof as follows: In the notation of [LT], we choose the family $\mathscr{V}$ in such a way that $\cup \hat{\mathscr{V}}^{\circ} \subset B^{\prime}$, where $\hat{\mathscr{V}}=\{V \in \mathscr{V} \mid V \cap B \neq \emptyset\}$. In the construction of the sets $C^{i}$ and the immersions $f_{i}$ we then replace $\mathscr{V}$ by $\hat{\mathscr{V}}$ and $\mathscr{V}_{i}$ by $\mathscr{V}_{i} \cap \hat{\mathscr{V}}$ for $1 \leqq i \leqq k$.

Theorem 5.3 for $C \neq$ TOP now follows from 4.1 and 5.1 as in 5.5 for $C=\mathrm{TOP}$.

5.10. Theorem. Let $C \neq \mathrm{TOP}, n, M, Q, A$ be as in 5.1, let $f: M \rightarrow Q$ be a locally flat embedding which is locally $C$-flat near $A$, and let $\varepsilon \in C_{+}(Q)$. Then there is an $\varepsilon$-push $h$ of $(Q, f[M \backslash A])$ such that $h f=f$ near $A$ and $h f$ is locally C-flat.

Proof. Choose open sets $U, V \subset M$ such that $A \subset V \subset \bar{V} \subset U$ and $f \mid U$ is locally $C$-flat. By 5.1 for every $\delta \in C_{+}(M)$ there is a locally $C$-flat embedding $g: M \rightarrow Q$ in $U(f, \delta)$ with $g|V=f| V$. By 4.1 there is an $\varepsilon$-push $h$ of $(Q, f[M \backslash A])$ such that $h f=g$, provided that $\delta$ is small enough. Then $h$ is the desired homeomorphism.

5.11. Theorem. Let $C \in\{\mathrm{LQC}, \mathrm{LIP}\}$ and $n \neq 4,5,6$, let $M$ be an $(n-1)$ and $Q$ an n-manifold with $\partial Q=\emptyset$, and let $f: M \rightarrow Q$ be a locally flat embedding. Then $M$ and $Q$ can be given $C$-structures such that $f$ becomes locally $C$-flat.

Moreover, if $M$ (or $\partial M$, respectively) is already a $C$-manifold, this can be done without changing the C-structure of $M$ (of $\partial M$, respectively) for all $n \neq 4$ ( $n \neq 4,5$, respectively).

Proof. We may assume that $M$ and $Q$ are $C$-manifolds by $\left[\mathrm{TV}_{3}\right.$, Theorems 4.6 and 4.8] and, whenever $\partial M \neq \emptyset$ and the parenthetic assertion is considered, by 8.2 . Then 5.11 follows from 5.10 .

An application of 5.11 is given in 8.3.2. 


\section{Locally LIP flat approximation of quasisymmetric or bilipschitz embeddings}

In this section we prove the following two theorems concerning, respectively, quasisymmetric embeddings and bilipschitz embeddings.

6.1. Theorem. Let $n \neq 4$ and let $\eta: R_{+}^{1} \rightarrow R_{+}^{1}$ be a homeomorphism. Then there is a homeomorphism $\eta^{*}: R_{+}^{1} \rightarrow R_{+}^{1}$ with the following property: Let either $Y \subset X \subset R^{n-1}$ with $Y$ open in $R^{n-1}$ or $Y \subset X \subset R_{+}^{n-1}$ with $Y$ open in $R_{+}^{n-1}$, let $f: X \rightarrow R^{n}$ be an $\eta$-quasisymmetric embedding, and let $\varepsilon \in C_{+}(Y)$. Then there is an $\eta^{*}$-quasisymmetric embedding $f^{*}: X \rightarrow R^{n}$ such that

(1) $f^{*}|X \backslash Y=f| X \backslash Y$,

(2) $f^{*} \mid Y \in U(f \mid Y, \varepsilon)$,

(3) $f^{*} \mid Y$ is locally LIP flat.

6.2. Theorem. Let $n \neq 4$ and let $L \geqq 1$. Then there is $L^{*} \geqq 1$ with the following property: Let $X, Y, \varepsilon$ be as in 6.1 and let $f: X \rightarrow R^{n}$ be an L-bilipschitz embedding. Then there is an $L^{*}$-bilipschitz embedding $f^{*}: X \rightarrow R^{n}$ satisfying the conditions (1), (2), and (3) of 6.1.

6.3. Remarks. 1. Theorems 6.1 and 6.2 are trivial for $n=1$. They are new for $n \geqq 5$ only because of their analogues mentioned in the Introduction and proved in [LT, Corollary 2.20 and Theorem 3.2]. In the proofs for $n \geqq 2$ we closely follow [LT]; we use Carleson's finiteness idea [Ca] (also used in $\left[\mathrm{V}_{2}\right],\left[\mathrm{T}_{1}\right],\left[\mathrm{T}_{2}\right]$, $\left.[\mathrm{Kk}],\left[\mathrm{TV}_{2}\right],\left[\mathrm{TV}_{3}\right]\right)$ but replace the PL methods of [LT] by 5.3 for LIP. As in [LT], in 6.1 and 6.2 we could suppose about $(X, Y)$ more generally that $X \subset R^{n-1}$ and that $Y$ is only an open subset of $X$ which is the union of a subfamily, locally finite in $Y$, of the cube family $\mathscr{L}$ of 6.4 and which is a manifold.

2. One obtains easily an analogue of 6.1 and 6.2 for weakly quasisymmetric embeddings, defined in $\left[\mathrm{TV}_{1}\right]$; cf. [LT, Theorem 2.22].

6.4. Cubical decompositions. Let $n \geqq 2$, let $Y$ be an open subset of $R^{n-1}$ or of $R_{+}^{n-1}$, let $\varphi: Y \rightarrow Z$ be a continuous map into a metric space, and let $\varepsilon \in C_{+}(Y)$. We define a decomposition $\mathscr{K}$ of $Y$ into closed $(n-1)$-cubes as follows. Let $\mathscr{L}$ be the family of all closed $(n-1)$-cubes in $R^{n-1}$ with side length $2^{-k}$ and with vertices in $2^{-k} \mathbf{Z}^{n-1}, k \in \mathbf{N}$. For each $Q \in \mathscr{L}$, we let $z_{Q}$ be its center and $2 \lambda_{Q}$ its side length. Define $\alpha_{Q}: R^{n-1} \rightarrow R^{n-1}$ by $\alpha_{Q}(x)=z_{Q}+\lambda_{Q} x$, and let $Q(t)=\alpha_{Q} J^{n-1}(t), \bar{Q}(t)=\overline{Q(t)}$ for $t>0$; then $Q=\bar{Q}(1)$. Let $\mathscr{K}^{0}$ be the family of all maximal cubes $Q$ of $\mathscr{L}$ contained in $Y$ and satisfying either the conditions $\bar{Q}(3) \subset Y$ and $d(\varphi \bar{Q}(3))<$ $\min \varepsilon \bar{Q}(3)$ whenever $Q \cap \partial Y=\emptyset$ or the conditions $\bar{Q}(3) \cap R_{+}^{n-1} \subset Y$ and $d\left(\varphi\left[\bar{Q}(3) \cap R_{+}^{n-1}\right]\right)<\min \varepsilon\left[\bar{Q}(3) \cap R_{+}^{n-1}\right]$ whenever $Q \cap \partial Y \neq \emptyset$. Dividing each cube of $\mathscr{K}^{0}$ into $N=2^{n-1}$ cubes by bisecting the sides we obtain the family $\mathscr{K}$. We express $\mathscr{K}$ as a disjoint union $\mathscr{K}=\mathscr{K}_{1} \cup \ldots \cup \mathscr{K}_{N}$ as follows: Divide $I^{n-1}$ into cubes 
$Q_{1}, \ldots, Q_{N}$ by bisecting the sides. Then set $\mathscr{K}_{i}=\left\{\alpha_{Q} Q_{i} \mid Q \in \mathscr{K}^{0}\right\}$. The following properties of $\mathscr{K}$ are easily verified:

(1) $Y=\cup \mathscr{K}$.

(2) If $Q, R \in \mathscr{K}, \quad Q \cap R \neq \emptyset, \quad$ and $\quad Q \neq R$, then int $Q$ คint $R=\emptyset \quad$ and $\lambda_{Q} / \lambda_{R} \in\{1 / 2,1,2\}$.

(3) If $Q \in \mathscr{K}$ and $Q \cap \partial Y=\emptyset$, then $\bar{Q}(3) \subset Y$. If $Q \in \mathscr{K}$ and $Q \cap \partial Y \neq \emptyset$, then $\bar{Q}(3) \cap R_{+}^{n-1} \subset Y$.

(4) If $Q, R \in \mathscr{K}$ and $Q \cap R=\emptyset$, then $Q(3 / 2) \cap R(3 / 2)=\emptyset$.

(5) If $x \in Q \in \mathscr{K}$, then $d(\varphi Q)<\varepsilon(x)$.

(6) If $Q, R \in \mathscr{K}_{i}$ and $Q \neq R$, then $Q \cap R=\emptyset$.

We call $\mathscr{K}$ the cubical $(\varphi, \varepsilon)$-decomposition of $Y$ or also a cubical decomposition of $Y$ if we do not specify $\varphi$ and $\varepsilon$.

6.5. From now on to the end of the proof of Lemma 6.13, we suppose that $n, \eta, X, Y, f$ are as in 6.1 with $n \geqq 2$, that $\mathscr{K}$ is a cubical decomposition of $Y$, and that $\sigma>0$. We may assume card $X \geqq 2$; then $\eta(1) \geqq 1$.

For each $Q \in \mathscr{K}$ we set $\varrho_{Q}=\left|f\left(z_{Q}\right)-f\left(z_{Q}+\lambda_{Q} e_{1}\right)\right|$ and $Q^{*}(t)=Q(t) \cap Y$ $(=Q(t) \cap X)$ for $t \in(0,3]$; then $\alpha_{Q}^{-1} Q^{*}(t)=J^{n-1}(t)$ if $Q \cap \partial Y=\emptyset$ and $\alpha_{Q}^{-1} Q^{*}(t)=$ $J^{n-1}(t) \cap\left(R_{+}^{n-1}-e_{n-1}\right)$ if $Q \cap \partial Y \neq \emptyset$.

The following lemma can be proved in the same way as [LT, Lemma 2.6].

6.6. Lemma. There are constants $c_{1} \geqq 1$ and $c_{2} \geqq 1$ depending only on $n$ and $\eta$ with the following properties:

(1) If $Q, R \in \mathscr{K}, Q \cap R \neq \emptyset$, and $Q \neq R$, then $\varrho_{Q} / c_{1} \leqq\left|f\left(z_{Q}\right)-f\left(z_{R}\right)\right| \leqq c_{1} \varrho_{Q}$.

(2) If $Q, R \in \mathscr{K}$ and $Q \cap R \neq \emptyset$, then $\varrho_{Q} / \varrho_{R} \leqq c_{1}^{2}$.

(3) If $Q \in \mathscr{K}, x \in Q^{*}(5 / 4)$, and $y \in X \backslash Q^{*}(4 / 3)$, then $|f(x)-f(y)| \geqq \varrho_{Q} / c_{2}$.

6.7. Constructions. Let $\varkappa_{1}, \varkappa_{2}$ be the least natural numbers such that $2^{\varkappa_{1}} \geqq 2 \sqrt{n}$ and $2^{x_{2}} \geqq 2 c_{1}^{2}$. For each $Q \in \mathscr{K}$ let $\mu_{Q}$ be the greatest integer such that $s_{Q}=2^{\mu_{Q}} \leqq \varrho_{Q}$.

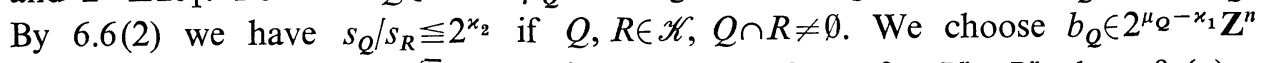
such that $\left|b_{Q}-f\left(z_{Q}\right)\right| \leqq \sqrt{n} 2^{\mu_{Q}-x_{1}-1}\left(\leqq s_{Q} / 4\right)$. Define $\beta_{Q}: R^{n} \rightarrow R^{n}$ by $\beta_{Q}(x)=$ $\left(x-b_{Q}\right) / s_{Q}$.

6.8. Lemma. There are a finite set $\mathscr{A}$ depending only on $n$ and a finite set $\mathscr{B}$ depending only on $n$ and $\eta$ such that if $Q, R \in \mathscr{K}, Q \cap R \neq \emptyset$, then $\alpha_{R}^{-1} \alpha_{Q} \in \mathscr{A}$ and $\beta_{Q} \beta_{\boldsymbol{R}}^{-1} \in \mathscr{B}$.

Proof. The existence of $\mathscr{A}$ follows from 6.4(2). The existence of $\mathscr{B}$ follows from an obvious analogue of [LT, Lemma 2.8], which can be proved by the aid of 6.6(1) and 6.6(2) exactly as [Kk, Lemma 2.4$]$. 
6.9. Constructions. We set for $0 \leqq i \leqq N$

$$
\begin{aligned}
& \mathscr{K}_{i}^{*}=\mathscr{K}_{1} \cup \ldots \cup \mathscr{K}_{i}, \\
& U_{i}=\cup\left\{Q^{*}\left(1+2^{-i-1}\right) \mid Q \in \mathscr{K}_{i}^{*}\right\}, \\
& V_{i}=\cup\left\{Q^{*}\left(1+2^{-i-2}\right) \mid Q \in \mathscr{K}_{i}^{*}\right\} .
\end{aligned}
$$

Then $U_{i}, V_{i}$ are open in $Y, V_{i-1} \subset U_{i}$, and $U_{N}=Y$. If $i \geqq 1$ and $Q \in \mathscr{K}_{i}$, we set

$$
\begin{aligned}
U_{Q} & =\alpha_{Q}^{-1}\left[Q^{*}(3 / 2) \cap U_{i-1}\right], \\
V_{Q} & =\alpha_{Q}^{-1}\left[Q^{*}(4 / 3) \cap V_{i-1}\right] .
\end{aligned}
$$

Then $\bar{V}_{Q} \subset U_{Q}$. There is a finite set $\mathscr{S}$ depending only on $n$ such that $\left(U_{Q}, V_{Q}\right) \in \mathscr{Y}$.

Let $\mathscr{T}=\left\{I^{n-1}(2), I^{n-1}(2) \cap\left(R_{+}^{n-1}-e_{n-1}\right)\right\}$. For $T \in \mathscr{T}$ let $\mathscr{F}_{\eta}(T)$ be the set of all $\eta$-quasisymmetric embeddings $g: T \rightarrow R^{n}$ with $|g(0)| \leqq 1 / 4$ and $3 / 4 \leqq\left|g\left(e_{1}\right)\right| \leqq 3$. In the topology of uniform convergence $\mathscr{F}_{\eta}(T)$ is compact by $\left[\mathrm{TV}_{1}\right.$, Theorems 3.4 and 3.7 and Remark 3.6]. For each $Q \in \mathscr{K}$ we set $T_{Q}=\alpha_{Q}^{-1}[\bar{Q}(2) \cap Y]$ and $f_{Q}=\beta_{Q} f \alpha_{Q} \mid T_{Q}$. Clearly $T_{Q} \in \mathscr{T}$ and $f_{Q} \in \mathscr{F}_{\eta}\left(T_{Q}\right)$.

6.10. Lemma. For every $\varepsilon^{\prime}>0$ there is $\delta^{*}\left(\varepsilon^{\prime}\right) \in\left(0, \varepsilon^{\prime}\right]$ depending only on $n, \eta$, and $\varepsilon^{\prime}$ which has the following property: If $\mathscr{K}$ is as above, $Q \in \mathscr{K}, g \in \mathscr{F}_{\eta}\left(T_{Q}\right)$, and if $h: U_{Q} \rightarrow R^{n}$ is a locally LIP flat embedding with $d\left(h, g \mid U_{Q}\right)<\delta^{*}\left(\varepsilon^{\prime}\right)$, there is a locally LIP flat embedding $h^{\prime}: T_{Q \rightarrow R^{n}}$ such that $d\left(h^{\prime}, g\right)<\varepsilon^{\prime}$ and $h^{\prime}\left|V_{Q}=h\right| V_{Q}$.

Proof. Consider $T \in \mathscr{T}$, an arbitrary embedding $g: T \rightarrow R^{n}$, and $\varepsilon^{\prime}>0$. Given $\mathscr{K}$ and $Q \in \mathscr{K}$ with $T_{Q}=T$, by 5.3 there is $\delta>0$ such that for every locally LIP flat embedding $h: U_{Q} \rightarrow R^{n}$ with $d\left(h, g \mid U_{Q}\right)<\delta$ there is $h^{\prime}$ as in the lemma. Since $\mathscr{S}$ is finite, there is the greatest $\delta=\delta\left(T, g, \varepsilon^{\prime}\right) \in\left(0, \varepsilon^{\prime}\right]$ having this property for all $\mathscr{K}$ and $Q \in \mathscr{K}$ with $T_{Q}=T$.

We set $\delta^{*}\left(\varepsilon^{\prime}\right)=\inf \left\{\delta\left(T, g, \varepsilon^{\prime}\right) \mid T \in \mathscr{T}, g \in \mathscr{F}_{\eta}(T)\right\}$. Then as in [ $\mathrm{V}_{2}$, Lemma 2.6] we get $\delta^{*}\left(\varepsilon^{\prime}\right)>0$ by the compactness of the spaces $\mathscr{F}_{\eta}(T)$. Thus $\delta^{*}\left(\varepsilon^{\prime}\right)$ is the desired number.

6.11. Construction. We choose numbers $\delta_{N} \geqq \delta_{N-1} \geqq \ldots \geqq \delta_{0}>0$ such that

$$
\begin{aligned}
& \delta_{N}<\min \left(\sigma / 2,1 / 4 c_{1}^{2} c_{2}, 1 / 4 c_{1}^{2} \eta(2) \eta(2 \sqrt{n-1})\right. \\
& \delta_{i}<\delta^{*}\left(\delta_{i+1}\right) /\left(2^{x_{2}+1}+1\right) .
\end{aligned}
$$

The numbers $\delta_{i}$ depend only on $n, \eta$, and $\sigma$.

We next prove by induction on $i$ that the following lemma is true for every $i \in\{0,1, \ldots, N\}$ :

$6.12_{i}$. Lemma. There are a finite set $\mathscr{H}_{i}$, depending only on $(n, \eta, \sigma, i)$, of locally LIP flat embeddings $J^{n-1}\left(1+2^{-i-1}\right) \cap T \rightarrow R^{n}, T \in \mathscr{T}$, and a locally LIP flat embedding $\varphi_{i}: U_{i} \rightarrow R^{n}$, such that for each $Q \in \mathscr{K}_{i}^{*}$

(1) $\beta_{Q} \varphi_{i} \alpha_{Q} \mid \alpha_{Q}^{-1} Q^{*}\left(1+2^{-i-1}\right) \in \mathscr{H}_{i}$,

(2) $d\left(\varphi_{i}\left|Q^{*}\left(1+2^{-i-1}\right), f\right| Q^{*}\left(1+2^{-i-1}\right)\right) \leqq 2 \delta_{i} s_{Q}$. 
Proof. Since $U_{0}=\emptyset, 6.12_{0}$ is trivially true. Assume that $1 \leqq i \leqq N$ and that $6.12_{i-1}$ is true. For $Q \in \mathscr{K}_{i}$ we define a locally LIP flat embedding $h_{Q}=\beta_{Q} \varphi_{i-1} \alpha_{Q} \mid U_{Q}$. If $x \in U_{Q}$, there is $R \in \mathscr{K}_{i-1}^{*}$ such that $\alpha_{Q}(x) \in R^{*}\left(1+2^{-i}\right)$, whence $R \cap Q \neq \emptyset$. Then $h_{Q}(x)=\beta_{Q} \beta_{R}^{-1} \psi \alpha_{R}^{-1} \alpha_{Q}(x)$, where $\quad \psi=\beta_{R} \varphi_{i-1} \alpha_{R} \mid \alpha_{R}^{-1} R^{*}\left(1+2^{-i}\right) \in \mathscr{H}_{i-1}$. The sets $U_{Q} \cap \alpha_{Q}^{-1} R^{*}\left(1+2^{-i}\right)$ belong to a finite set depending only on $n$. Thus by 6.8 the maps $h_{Q}$ belong to a finite set $\mathscr{C}_{i}$ depending only on $(n, \eta, \sigma, i)$.

For each $T \in \mathscr{T}$, by the compactness of $\mathscr{F}_{\eta}(T)$ there is a finite set $\mathscr{F}^{\prime}(T) \subset \mathscr{F}_{\eta}(T)$ depending only on $(n, \eta, \sigma, i)$ such that $d\left(g, \mathscr{F}^{\prime}(T)\right)<\delta_{i-1}$ if $g \in \mathscr{F}_{\eta}(T)$. Consider $Q \in \mathscr{K}_{i}$. Choose $g \in \mathscr{F}^{\prime}\left(T_{Q}\right)$ with $d\left(f_{Q}, g\right)<\delta_{i-1}$. If $x \in U_{Q}$ and $R$ is as above,

$$
\begin{aligned}
\left|h_{Q}(x)-f_{Q}(x)\right| & =\left|\varphi_{i-1}\left(\alpha_{Q}(x)\right)-f\left(\alpha_{Q}(x)\right)\right| / s_{Q} \\
& \leqq 2 \delta_{i-1} s_{R} / s_{Q} \leqq 2^{x_{2}+1} \delta_{i-1},
\end{aligned}
$$

whence $d\left(h_{Q}, g \mid U_{Q}\right)<\delta^{*}\left(\delta_{i}\right)$. Hence there is a locally LIP flat embedding $h_{Q}^{\prime}: T_{Q} \rightarrow R^{n}$ such that $d\left(h_{Q}^{\prime}, g\right)<\delta_{i}$ and $h_{Q}^{\prime}=h_{Q}$ on $V_{Q}$. We can choose the maps $h_{Q}^{\prime}$ in such a way that the maps $h_{Q}^{\prime} \mid \alpha_{Q}^{-1} Q^{*}\left(1+2^{-i-1}\right)$ belong to a finite set $\mathscr{D}_{i}$, depending only on $(n, \eta, \sigma, i)$, of locally LIP flat embeddings $J^{n-1}\left(1+2^{-i-1}\right) \cap T \rightarrow R^{n}, T \in \mathscr{T}$.

We set $\varphi_{i}\left|V_{i-1}=\varphi_{i-1}\right| V_{i-1}$ and $\varphi_{i}(x)=\beta_{Q}^{-1} h_{Q}^{\prime} \alpha_{Q}^{-1}(x)$ for all $Q \in \mathscr{K}_{i}$ and $x \in Q^{*}\left(1+2^{-i-1}\right)$. Then $\varphi_{i}: U_{i} \rightarrow R^{n}$ is a well-defined locally LIP flat immersion, and it satisfies (1) with

$$
\mathscr{H}_{i}=\mathscr{D}_{i} \cup\left\{h\left|J^{n-1}\left(1+2^{-i-1}\right) \cap T\right| h: J^{n-1}\left(1+2^{-i}\right) \cap T \rightarrow R^{n}, T \in \mathscr{T}, h \in \mathscr{H}_{i-1}\right\} .
$$

If $Q \in \mathscr{K}_{i}$ and $x \in Q^{*}\left(1+2^{-i-1}\right)$,

$$
\left|\varphi_{i}(x)-f(x)\right|=s_{Q}\left|h_{Q}^{\prime}\left(\alpha_{Q}^{-1}(x)\right)-f_{Q}\left(\alpha_{Q}^{-1}(x)\right)\right| \leqq 2 \delta_{i} s_{Q},
$$

which implies (2). We finally prove that $\varphi_{i}$ is an embedding. Since $\varphi_{i}$ is the embedding $\beta_{Q}^{-1} h_{Q}^{\prime} \alpha_{Q}^{-1}$ on $Q^{*}(4 / 3) \cap U_{i}$ for each $Q \in \mathscr{K}_{i}$, it suffices to show that if $Q, R \in \mathscr{K}_{i}^{*}, \quad x \in Q^{*}\left(1+2^{-i-1}\right), \quad y \in R^{*}\left(1+2^{-i-1}\right), \quad s_{Q} \geqq s_{R}, \quad \Delta=\left|\varphi_{i}(x)-\varphi_{i}(y)\right|, \quad$ and if $x \notin R^{*}(4 / 3)$ or $y \notin Q^{*}(4 / 3)$, then $\Delta \geqq \lambda s_{Q}$, where $\lambda=1 / c_{1}^{2} c_{2}-4 \delta_{i}>0$. We have $\Delta \geqq|f(x)-f(y)|-4 \delta_{i} s_{Q}$. Thus, if $y \notin Q^{*}(4 / 3)$, the claim follows from 6.6(3), whereas if $y \in Q^{*}(4 / 3)$, in which case $Q \cap R \neq \emptyset$ and $x \notin R^{*}(4 / 3)$, the claim follows from 6.6(3) and 6.6(2).

6.13. Lemma. Let $2 \leqq n \neq 4$, let $\eta: R_{+}^{1} \rightarrow R_{+}^{1}$ be a homeomorphism, and let $\sigma>0$. Then there are a homeomorphism $\eta^{*}: R_{+}^{1} \rightarrow R_{+}^{1}$ and a finite set $\mathscr{D}$ of locally LIP flat embeddings $T \rightarrow R^{n}, T \in \mathscr{T}$, with the following property: Let either $Y \subset X \subset$ $R^{n-1}$ with $Y$ open in $R^{n-1}$ or $Y \subset X \subset R_{+}^{n-1}$ with $Y$ open in $R_{+}^{n-1}$, let $f: X \rightarrow R^{n}$ be an $\eta$-quasisymmetric embedding, and let $\mathscr{K}$ be a cubical decomposition of $Y$. Then there is an $\eta^{*}$-quasisymmetric embedding $f^{*}: X \rightarrow R^{n}$ such that

(1) $f^{*}|X \backslash Y=f| X \backslash Y$,

(2) $f^{*} \mid Y$ is locally LIP flat,

(3) $d\left(f^{*}|Q, f| Q\right) \leqq \sigma \varrho_{Q}$ for each $Q \in \mathscr{K}$,

(4) $\beta_{Q} f^{*} \alpha_{Q} \mid T_{Q} \in \mathscr{D}$ for each $Q \in \mathscr{K}$. 
Proof. Let $\varphi_{N}: Y \rightarrow R^{n}$ be the locally LIP flat embedding of $6.12_{N}$. We define $f^{*}=\varphi_{N} \cup(f \mid X \backslash Y)$. Then (1), (2), and (3) are satisfied. One can find $\mathscr{D}$ and prove (4) in the same way as one obtained $\mathscr{C}_{i}$ and the relation $h_{Q} \in \mathscr{C}_{i}$ in the proof of $6.12_{i}$. The proof of [LT, Theorem 2.16] now shows that $f^{*}$ is an $\eta^{*}$-quasisymmetric embedding with $\eta^{*}$ depending only on $(n, \eta, \sigma)$.

6.14. Proof of 6.1 for $n \geqq 2$. Apply 6.13 with $\sigma=1$ and with $\mathscr{K}$ being the cubical $(f, \varepsilon)$-decomposition of $Y$.

6.15. Proof of 6.2 for $n \geqq 2$. Every $L$-bilipschitz embedding is $\eta_{L}$-quasisymmetric, $\eta_{L}(t)=L^{2} t$. Theorem 6.2 follows from an application of 6.13 with the substitution $\eta \mapsto \eta_{L}, \sigma \mapsto 1 / 3 L^{2}$. The details are almost the same as those of the proof of [LT, Theorem 3.2].

\section{Relative LQS or LIP approximation}

In this section we prove the following two theorems dealing with relative LQS or LIP approximation of embeddings or of immersions, respectively. Their essence is the special case $A \neq \emptyset, U=B=B^{\prime}=M$.

7.1. Theorem. Let $C \in\{\mathrm{LQS}, \mathrm{LIP}\}$ and $n \neq 4$, let $M$ be an $(n-1)$ - and $Q$ an n-dimensional $C$-manifold with $\partial Q=\emptyset$, let $A \subset M$ be closed and $U, B \subset M$ open with $A \subset U$, let $f: M \rightarrow Q$ be an embedding which is a C-embedding near $A$, and let $\varepsilon \in C_{+}(B)$. Then there is an embedding $f^{*}: M \rightarrow Q$ such that $f^{*}=f$ near $A$ and on $M \backslash B$, that $f^{*} \mid B \in U(f \mid B, \varepsilon)$, that $f^{*}$ is a $C$-embedding near $A \cup B$, and that $f^{*}$ is locally $C$-flat near $B \backslash U$.

7.2. Theorem. Let $C, n, M, Q, A, U$ be as in 7.1, let $B \subset M$ be closed and $B^{\prime} \subset M$ open with $B \subset B^{\prime}$, let $f: M \rightarrow Q$ be an immersion which is a $C$-immersion near $A$, and let $\varepsilon \in C_{+}\left(B^{\prime}\right)$. Then there is an immersion $f^{*}: M \rightarrow Q$ such that $f^{*}=f$ near $A$ and on $M \backslash B^{\prime}$, that $f^{*} \mid B^{\prime} \in U\left(f \mid B^{\prime}, \varepsilon\right)$, that $f^{*}$ is a $C$-immersion near $A \cup B$, and that $f^{*}$ is locally $C$-flat near $B \backslash U$.

We begin with an analogue of [LT, Lemma 4.2] and of 5.8.

7.3. Lemma. Let $C \in\{\mathrm{LQS}, \mathrm{LIP}\}$ and $n \neq 4$, let $B \subset A$ be open subsets of $R_{+}^{n-1}$ such that $\bar{B} \subset A$ is compact, let $f: A \rightarrow R^{n}$ be an embedding which is a $C$ embedding near a closed subset $E$ of $A$, and let $\varepsilon>0$. Then there is an embedding $f^{*}: A \rightarrow R^{n}$ such that $f^{*}=f$ on $A \backslash B, d\left(f^{*}, f\right)<\varepsilon$, and $f^{*}$ is a $C$-embedding near $E \cup B$.

Proof. We may assume that $E$ is compact. Define $v \in C_{+}(B)$ by $v(x)=$ $\min (\varepsilon, d(f(x), f[A \backslash B]) / 2)$. Choose an open neighborhood $X$ of $E$ in $A$ such that $\bar{X} \subset A$ is compact and $f \mid \bar{X}$ is a $C$-embedding. Then $f \mid X$ is quasisymmetric 
if $C=\mathrm{LQS}$ or bilipschitz if $C=\mathrm{LIP}$. Let $Y=X \cap B$, and define $\delta \in C_{+}(Y)$ by $\delta(x)=(1 / 2) \min (v(x), d(f(x), f[B \backslash Y]))$. By 6.1 if $C=\mathrm{LQS}$ or by 6.2 if $C=\mathrm{LIP}$, there is a $C$-embedding $g: X \rightarrow R^{n}$ such that $g=f$ on $X \backslash Y, g \mid Y \in U(f \mid Y, \delta)$, and $g \mid Y$ is locally LIP flat. Extending $g \mid Y$ by $f \mid B \backslash Y$ we obtain an embedding $g_{1}: B \rightarrow R^{n}$.

Choose an open neighborhood $V$ of $E$ in $A$ with $\bar{V} \subset X$. Since $g_{1}$ is locally LIP flat on $Y \supset \bar{V} \cap B$, by 5.1 there is a locally LIP flat embedding $f_{1}: B \rightarrow R^{n}$ in $U\left(g_{1}, v / 2\right)$ such that $f_{1}=g_{1}$ on $V \cap B$. Then $f_{1} \in U(f \mid B, v)$. Thus $f_{1}$ and $f \mid A \backslash B$ define an embedding $f^{*}: A \rightarrow R^{n}$, which is the desired one since $f^{*}=g$ on $V$.

7.4. Lemma. Theorem 7.1 holds if $U=B=M$, and Theorem 7.2 holds if $U=M$.

Proof. This follows from 7.3 in the same way as 5.1 and 5.2 followed from 5.8 in 5.9.

7.5. Proof of 7.2. Choose an open $V \subset M$ with $B \backslash U \subset V \subset \bar{V} \subset B^{\prime} \backslash A$. By 5.2 there is an immersion $g: M \rightarrow Q$ such that $g=f$ on $M \backslash V, g \mid V \in U(f|V,(\varepsilon / 2)| V)$ and $g$ is locally $C$-flat near $B \backslash U$. Then $g=f$ near $A$, whence $g$ is a $C$-immersion near $A \cup(B \backslash U)$. Thus by 7.4 there is an immersion $f^{*}: M \rightarrow Q$ such that $f^{*}=g$ near $A \cup(B \backslash U)$ and on $M \backslash B^{\prime}, f^{*} \mid B^{\prime} \in U\left(g \mid B^{\prime}, \varepsilon / 2\right)$, and $f^{*}$ is a $C$-immersion near $A \cup B$. Then $f^{*}$ is the desired map.

7.6. Lemma. Theorem 7.1 holds if $B=M$.

Proof. This reduces to 7.4 by 5.1 ; the proof is similar to 7.5 .

7.7. Proof of 7.1. We may assume that $\varepsilon(x) \leqq d(f(x), f[M \backslash B]) / 2$ for each $x \in B$. Choose an open $V \subset M$ such that $A \subset V \subset \bar{V} \subset U$ and that $f$ is a $C$-embedding near $\bar{V}$. By 7.6 there is a $C$-embedding $g: B \rightarrow Q$ in $U(f \mid B, \varepsilon)$ such that $g=f$ on $V \cap B$ and $g$ is locally $C$-flat near $B \backslash U$. Then $f^{*}=g \cup(f \mid M \backslash B)$ is the desired embedding since $f^{*}=f$ on $V$.

\section{Extension of LQC or LIP structures}

In this section $C \in\{\mathrm{LQC}, \mathrm{LIP}\}, M$ is an $n$-manifold, and $\mathscr{A}$ is a $C$-structure on $\partial M$. We prove the following two theorems.

8.1. Theorem. Suppose that $M$ is of the form $M=\partial M \times R_{+}^{1}$. Then $\mathscr{A}$ can be extended to a $C$-structure on $M$.

8.2. Theorem. Suppose $n \neq 4$. Then $\mathscr{A}$ can be extended to a $C$-structure on $M$. 
Moreover, if $\mathscr{A}_{1}$ and $\mathscr{A}_{2}$ are two $C$-structures on $M$ both extending $\mathscr{A}$, then for every $\varepsilon \in C_{+}(M)$ there is an ambient $\varepsilon$-isotopy $H$ of $M$ rel $\partial M$ such that $H_{1}:\left(M, \mathscr{A}_{1}\right) \rightarrow\left(M, \mathscr{A}_{2}\right)$ is a C-homeomorphism.

8.3. Remarks. 1. By [LT, Remark 4.7] every LQC manifold $(N, \mathscr{B})$ has a metric $\varrho$ such that id: $(N, \mathscr{B}) \rightarrow(N, \varrho)$ is an LQS homeomorphism. In 8.1 for $C=\mathrm{LQC}, n=2,\left[\mathrm{~T}_{3}\right.$, Example 4] shows that if $(\partial M, \mathscr{A})$ is given such a suitable metric, a product metric is not necessarily a suitable one for the extended LQC structure on $M$.

2. One gets the existence part of 8.2 immediately from 5.11 applying it to the inclusion of $(\partial M, \mathscr{A})$ into the double of $M$. However, we give below a more elementary proof.

8.4. Proof of 8.1 and 8.2. The uniqueness part of 8.2 follows immediately from $\left[\mathrm{TV}_{3}\right.$, Theorem 4.8]. Since $\partial M$ has a collar in $M$, it is easy to see by $\left[\mathrm{TV}_{3}\right.$, Theorem 4.6 and Corollary 4.5] that the existence part of 8.2 follows from 8.1. Theorem 8.1 is trivial for LIP. For $n \neq 5$, the LQC case of 8.1 follows from the LIP case, because the LQC structure $\mathscr{A}$ contains a LIP structure by $\left[\mathrm{TV}_{3}\right.$, Theorem 4.6 and Corollary 4.5]. Thus it suffices to prove 8.1 for $C=\mathrm{LQC}, n=5$. Since $(\partial M, \mathscr{A})$ has an open cover by $n$ sets each $C$-embeddable into $R^{n-1}$ (cf. [Mu, Lemma 2.7]), the following lemma completes the proof.

8.5. Lemma. Let $M$ be as in 8.1 with $n \neq 4$, write $X^{\prime}=X \times R_{+}^{1}$ whenever $X \subset \partial M \equiv \partial M \times\{0\}$, let $U, V$ be open subsets of $\partial M$, let $\mathscr{A}_{1}$ be a $C$-structure on $U^{\prime}$ with $\mathscr{A}_{1}|U=\mathscr{A}| U$, and let $f:(V, \mathscr{A} \mid V) \rightarrow R^{n-1}$ be a C-embedding. Then there is a C-structure $\mathscr{A}_{2}$ on $(U \cup V)^{\prime}$ such that $\mathscr{A}_{2}|U \cup V=\mathscr{A}| U \cup V$ and $\mathscr{A}_{2} \mid U^{\prime}=\mathscr{A}_{1}$.

Proof. Let $\mathscr{A}_{3}$ be the unique $C$-structure on $V^{\prime}$ which makes the open embedding $f \times$ id: $V^{\prime} \rightarrow R_{+}^{n}$ a $C$-embedding. Then $\mathscr{A}_{3}|V=\mathscr{A}| V$. Let $W=U \cap V$. By the uniqueness part of 8.2 there is a $C$-homeomorphism $h_{0}:\left(W^{\prime}, \mathscr{A}_{1} \mid W^{\prime}\right) \rightarrow$ $\left(W^{\prime}, \mathscr{A}_{3} \mid W^{\prime}\right)$ which can be extended by the identity of $V^{\prime} \backslash W^{\prime}$ to a homeomorphism $h: V^{\prime} \rightarrow V^{\prime}$ such that $h \mid V=$ id. Let $\mathscr{A}_{4}$ be the unique $C$-structure on $V^{\prime}$ which makes $h:\left(V^{\prime}, \mathscr{A}_{4}\right) \rightarrow\left(V^{\prime}, \mathscr{A}_{3}\right) \quad$ a $C$-homeomorphism. Then $\mathscr{A}_{4} \mid V=\mathscr{A} V$ and $\mathscr{A}_{4}\left|W^{\prime}=\mathscr{A}_{1}\right| W^{\prime}$. Hence the $C$-structure $\mathscr{A}_{2}$ on $(U \cup V)^{\prime}$ induced by $\mathscr{A}_{1} \cup \mathscr{A}_{4}$ is the desired $C$-structure.

\section{Extension of LQC homeomorphisms}

In this section we prove that for every $n$, every LQC homeomorphism $f: R^{n} \rightarrow R^{n}$ can be extended to an LQC homeomorphism $F: R_{+}^{n+1} \rightarrow R_{+}^{n+1}$; cf. $\left[\mathrm{TV}_{3}\right.$, Theorem 4.10]. The proof makes use of the fundamental Theorem 3.2 of Tukia and Väisälä $\left[\mathrm{TV}_{2}\right]$ on similar problems for quasiconformal embeddings. For $n \neq 3$ the result is simple: $\left[\mathrm{TV}_{3}\right.$, Theorem 4.8] produces $F$ as an LQC approxima- 
tion of $f \times$ id. (In the case $n=3$, it seems that [Ca] does not suffice for us.) In 9.4 we give a short proof for a slightly improved version of $\left[G_{2}\right.$, Theorem 2].

9.1. Lemma. Let $f: R^{n} \rightarrow R^{n}(n \geqq 1)$ be an LQC homeomorphism and let $A \subset R_{+}^{n+1}$ be compact. Then there is $r_{0}>0$ such that for every $r \geqq r_{0}$ there is an embedding $g: R^{n} \cup B_{+}^{n+1}(r) \rightarrow R_{+}^{n+1}$ such that $g\left|R^{n}=f, g\right| B_{+}^{n+1}(r)$ is quasiconformal, and $g B_{+}^{n+1}\left(r_{0}\right) \supset A$.

Proof. We may assume that $f$ is sense-preserving. Choose consecutively $a, \varrho, r_{0}>0 \quad$ such that $A \subset B^{n}(a) \times[0, a), f^{-1} B^{n}(a) \subset B^{n}(\varrho), d\left(f B^{n}(\varrho)\right) \geqq 2 a, \quad$ and $B^{n}(\varrho) \times[0,2 \varrho) \subset B_{+}^{n+1}\left(r_{0}\right)$. We extend $f$ to a homeomorphism $F_{f}: R_{+}^{n+1} \rightarrow R_{+}^{n+1}$ by setting $F_{f}(x, t)=(f(x), \tau(x, t))$ for $x \in R^{n}, t \geqq 0$, where

$$
\tau(x, t)=\max \left\{|f(y)-f(x)|: y \in R^{n},|y-x| \leqq t\right\}
$$

(cf. $\left.\left[\mathrm{TV}_{2},(2.4)\right]\right)$. For each $x \in B^{n}(\varrho)$ we have $\tau(x, 2 \varrho) \geqq a$ since $B^{n}(x, 2 \varrho) \supset B^{n}(\varrho)$. Hence $F_{f} B_{+}^{n+1}\left(r_{0}\right) \supset A$. Consider $r \geqq r_{0}$. Let $b=9+5 \sqrt{n}, r^{\prime}=(1+b) r, U=B^{n}\left(r^{\prime}\right)$, and $U^{*}=\left\{(x, t) \in R_{+}^{n+1}:|x|+b t<r^{\prime}\right\}$. Then $U^{*} \cap R^{n}=U$ and $\bar{B}_{+}^{n+1}(r) \subset U^{*}$. Since $f \mid U$ is quasiconformal, by [ $\mathrm{TV}_{2}$, Theorem 3.2] there is a quasiconformal embedding $F: U^{*} \rightarrow R_{+}^{n+1}$ such that $F|U=f| U$ and $F B_{+}^{n+1}\left(r_{0}\right) \supset A$. Then $g=f \cup\left(F \mid B_{+}^{n+1}(r)\right)$ is the desired embedding.

9.2. Theorem. For every $n \geqq 0$, every LQC homeomorphism $f: R^{n} \rightarrow R^{n}$ can be extended to an LQC homeomorphism $F: R_{+}^{n+1} \rightarrow R_{+}^{n+1}$.

Proof. We may assume $n \geqq 1$. We construct inductively numbers $s_{j}>r_{j}>j$ and embeddings $g_{j}: R^{n} \cup B_{+}^{n+1}\left(s_{j}\right) \rightarrow R_{+}^{n+1}, j \in \mathrm{N}$, such that $r_{j}<r_{j+1}, g_{j} \mid R^{n}=f$, $g_{j} \mid B_{+}^{n+1}\left(s_{j}\right)$ is quasiconformal, $g_{j} B_{+}^{n+1}\left(r_{j}\right) \supset \bar{B}_{+}^{n+1}(j)$, and $g_{j+1}=g_{j}$ on $\bar{B}_{+}^{n+1}\left(r_{j}\right)$. We obtain $s_{1}, r_{1}, g_{1}$ from 9.1 .

Suppose that we have constructed $s_{j}, r_{j}, g_{j}$. Let $H=R_{+}^{n+1} \backslash B_{+}^{n+1}\left(r_{j}\right), H^{\prime}=$ $R_{+}^{n+1} \backslash g_{j} B_{+}^{n+1}\left(r_{j}\right)$. Clearly there is a quasiconformal homeomorphism $\alpha: R^{n+1} \rightarrow R^{n+1}$ with $\alpha H=R_{+}^{n+1}$. We next construct a quasiconformal homeomorphism $\beta: R^{n+1} \rightarrow R^{n+1}$ with $\beta H^{\prime}=R_{+}^{n+1}$. Extend $g_{j} \mid B_{+}^{n+1}\left(s_{j}\right)$ by reflection to a quasiconformal embedding $\varphi: B^{n+1}\left(s_{j}\right) \rightarrow R^{n+1}$. By [GV, Relative Schoenflies theorem 2.4] there is a quasiconformal homeomorphism $\psi: R^{n+1} \rightarrow R^{n+1}$ such that $\psi=\varphi$ on $\bar{B}^{n+1}\left(r_{j}\right)$ and $\psi R^{n}=R^{n}$. Then $\psi H=H^{\prime}$. Thus $\beta=\alpha \psi^{-1}$ is the desired map. Using the equivalent definition of LQC embeddings in a Euclidean space as LQS embeddings [ $\mathrm{V}_{3}$, Corollary 2.6], we see that the homeomorphism $h_{0}=\beta g_{j} \alpha^{-1}: R^{n} \rightarrow R^{n}$ is LQC. By 9.1 there is $r_{0}>0$ such that for every $r \geqq r_{0}$ there is an embedding $h: R^{n} \cup B_{+}^{n+1}(r) \rightarrow R_{+}^{n+1}$ such that $h\left|R^{n}=h_{0}, h\right| B_{+}^{n+1}(r)$ is quasiconformal, and $h B_{+}^{n+1}\left(r_{0}\right) \supset \beta\left[\bar{B}_{+}^{n+1}(j+1) \cap H^{\prime}\right]$. Choose $\quad r_{j+1}>\max \left(r_{j}, j+1\right) \quad$ such that $\alpha\left[B_{+}^{n+1}\left(r_{j+1}\right) \cap H\right] \supset B_{+}^{n+1}\left(r_{0}\right)$. Set $s_{j+1}=2 r_{j+1}$, and fix $r$ such that $\alpha\left[B_{+}^{n+1}\left(s_{j+1}\right) \cap H\right] \subset$ $B_{+}^{n+1}(r)$. Define an embedding $h_{1}:\left(R^{n} \cup B_{+}^{n+1}\left(s_{j+1}\right)\right) \cap H \rightarrow R_{+}^{n+1}$ by $h_{1}(x)=\beta^{-1} h \alpha(x)$. Then $h_{1}=g_{j}$ on $\partial H, h_{1} \mid B_{+}^{n+1}\left(s_{j+1}\right) \cap H$ is quasiconformal, and $h_{1}\left[B_{+}^{n+1}\left(r_{j+1}\right) \cap H\right] \supset$ 
$\bar{B}_{+}^{n+1}(j+1) \cap H^{\prime}$. Thus, extending $h_{1}$ by $g_{j} \mid B_{+}^{n+1}\left(r_{j}\right)$ we obtain the desired embedding $g_{j+1}$.

Setting $F=g_{j}$ on $B_{+}^{n+1}\left(r_{j}\right)$ for each $j \in \mathbf{N}$ we obtain an LQC homeomorphism $F: R_{+}^{n+1} \rightarrow R_{+}^{n+1}$ which extends $f$.

9.3. Corollary. For all $0 \leqq n \leqq p$, every LQC homeomorphism $f: R^{n} \rightarrow R^{n}$ can be extended to an LQC homeomorphism $F: R^{p} \rightarrow R^{p}$.

9.4. Theorem. Let $C \in\{\mathrm{LQC}, \mathrm{LIP}\}, n \neq 5$, and let $M_{1}, M_{2}$ be homeomorphic compact locally $C$-flat $(n-1)$-submanifolds of $S^{n}$ without boundary. Then there are open neighborhoods $U_{i}$ of $M_{i}$ in $S^{n}$ and a C-homeomorphism $f:\left(U_{1}, M_{1}\right) \rightarrow$ $\left(U_{2}, M_{2}\right)$.

Moreover, if $f_{0}: M_{1} \rightarrow M_{2}$ is a C-homeomorphism, this also holds for $n=5$, and we may assume $f \mid M_{1}=f_{0}$.

Proof. We may assume that $M_{1}, M_{2}$ are connected and $n \geqq 2$. By [Gr, Theorem 27.10], $S^{n} \backslash M_{i}$ consists of two components; their closures $M_{i 1}, M_{i 2}$ are $C$-manifolds with $\partial M_{i j}=M_{i}$. By [ $\mathrm{TV}_{3}$, Corollary 4.5] there is a $C$-homeo-

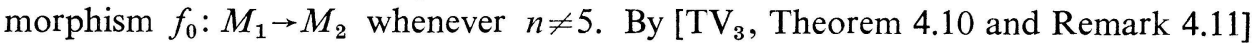
there are open neighborhoods $U_{i j}$ of $M_{i}$ in $M_{i j}$ and $C$-homeomorphisms $f_{j}: U_{1 j} \rightarrow U_{2 j}, i, j=1,2$, such that $f_{j} \mid M_{1}=f_{0}$. Then $U_{i}=U_{i 1} \cup U_{i 2}$ and $f=f_{1} \cup f_{2}$ satisfy the theorem.

\section{References}

[AC] ANCEL, F. D., and J. W. CANnON: The locally flat approximation of cell-like embedding relations. - Ann. of Math. (2) 109, 1979, 61-86.

[B $\left.{ }_{1}\right]$ BING, R. H.: Locally tame sets are tame. - Ibid. 59, 1954, 145-158.

[B $\left.B_{2}\right]$ BING, R. H.: Approximating surfaces with polyhedral ones. - Ibid. 65, 1957, 456-483.

[Ca] Carleson, L.: The extension problem for quasiconformal mappings. - Contributions to analysis, edited by L. V. Ahlfors et al., Academic Press, New York-London, 1974, $39-47$.

[Cr] Craggs, R.: Small ambient isotopies of a 3-manifold which transform one embedding of a polyhedron into another. - Fund. Math. 68, 1970, 225-256.

[D] Daverman, R. J.: Embeddings of $(n-1)$-spheres in Euclidean $n$-space. - Bull. Amer. Math. Soc. 84, 1978, 377-405.

[E] EDWARDs, R. D.: The equivalence of close piecewise linear embeddings. - General Topology Appl. 5, 1975, 147-180.

[G G GAULD, D.: Lipschitz and quasiconformal tubular neighbourhoods of spheres in codimension two. - Ann. Acad. Sci. Fenn. Ser. A I Math. 5, 1980, 175-178.

[G $\left.\mathrm{G}_{2}\right]$ GAULD, D.: Lipschitz and quasiconformal flattening of manifolds in codimension one. Univ. of Auckland, Dep. Math., Report Series 172, 1981, 1-8.

[GV] GAULD, D. B., and J. VÄrs̈̈LÄ: Lipschitz and quasiconformal flattening of spheres and cells. - Ann. Acad. Sci. Fenn. Ser. A I Math. 4, 1978/1979, 371-382. 
[Gr] Greenberg, M. J.: Lectures on algebraic topology. - W. A. Benjamin, Inc., New YorkAmsterdam, 1967.

[H] Hudson, J. F. P.: Piecewise linear topology. - W. A. Benjamin, Inc., New York-Amsterdam, 1969.

[Kk] KinkkA, M.: Piecewise linear approximation of quasiconformal and Lipschitz nomeomorphisms. - Ann. Acad. Sci. Fenn. Ser. A I Math. Dissertationes 30, 1980, 1-24.

[Ks] Kister, J. M.: Isotopies in 3-manifolds. - Trans. Amer. Math. Soc. 97, 1960, 213-224.

$\left[\mathrm{LV}_{1}\right]$ Lehto, O., and K. I. VIRTANEN: On the existence of quasiconformal mappings with prescribed complex dilatation. - Ann. Acad. Sci. Fenn. Ser. A I Math. 274, 1960, 1-24.

$\left[\mathrm{LV}_{2}\right]$ Lehto, O., and K. I. VIRTANEN: Quasiconformal mappings in the plane. - Die Grundlehren der mathematischen Wissenschaften in Einzeldarstellungen 126, Springer-Verlag, Berlin-Heidelberg-New York, 1973.

[LT] LUUKKAINEN, J., and P. TUKIA: Quasisymmetric and Lipschitz approximation of embeddings. - Ann. Acad. Sci. Fenn. Ser. A I Math. 6, 1981, 343-367.

[LuV] LUUKKAINEN, J., and J. VÄIsÄLÄ: Elements of Lipschitz topology. - Ibid. 3, 1977, 85-122.

[M] Matsumoto, Y.: Wild embeddings of piecewise linear manifolds in codimension two. Geometric topology (Proc. Georgia Topology Conf., Athens, Ga., 1977), edited by J. C. Cantrell, Academic Press, New York-San Francisco--London, 1979, 393-428.

[Ma] MatveEv, S. V.: Approximation of embeddings of manifolds by locally flat embeddings in codimension greater than 2. - Moscow Univ. Math. Bull. 27 (1972), 1973, 112-116.

[Mi] Miller, R. T.: Approximating codimension 3 embeddings. - Ann. of Math. (2) 95, 1972, 406-416.

[Mu] Munkres, J. R.: Elementary differential topology. - Annals of Mathematics Studies 54, Princeton University Press, Princeton, N. J., 1963.

[PS] Price, T. M., and C. L. SeebeCK III: Somewhere locally flat codimension one manifolds with 1-ULC complements are locally flat. - Trans. Amer. Math. Soc. 193, 1974, 111-122.

[Ri] Rickman, S.: Characterization of quasiconformal arcs. - Ann. Acad. Sci. Fenn. Ser. A I Math. 395, 1966, 1-30.

[RS] Rourke, C. P., and B. J. SANDERson: Introduction to piecewise-linear topology. - Ergebnisse der Mathematik und ihrer Grenzgebiete 69, Springer-Verlag, Berlin-HeidelbergNew York, 1972.

[Ru] Rushing, T. B.: Topological embeddings. - Pure and Applied Mathematics 52, Academic Press, New York-London, 1973.

[S] Sullivan, D.: Hyperbolic geometry and homeomorphisms. - Geometric topology (Proc. Georgia Topology Conf., Athens, Ga., 1977), edited by J. C. Cantrell, Academic Press, New York-San Francisco-London, 1979, 543--555.

$\left[T_{1}\right]$ TukiA, P.: Lipschitz approximation of homeomorphisms. - Ann. Acad. Sci. Fenn. Ser. A I Math. 4, 1978/1979, 137-144.

$\left[\mathrm{T}_{2}\right]$ TukIA, P.: The planar Schönflies theorem for Lipschitz maps. - Ibid. 5, 1980, 49-72.

$\left[\mathrm{T}_{3}\right]$ Tukia, P.: A quasiconformal group not isomorphic to a Möbius group. - Ibid. 6, 1981, $149-160$.

[TV $\left.{ }_{1}\right]$ TUKIA, P., and J. VÄISÄLÄ: Quasisymmetric embeddings of metric spaces. - Ibid. 5, 1980, 97-114.

[TV $\left.{ }_{2}\right]$ TUkıA, P., and J. VÄISÄLÄ: Quasiconformal extension from dimension $n$ to $n+1$. - Ann. of Math. (2) 115, 1982, 331-348.

$\left[\mathrm{TV}_{3}\right]$ TUkIA, P., and J. VÄISÄLÄ: Lipschitz and quasiconformal approximation and extension. Ann. Acad. Sci. Fenn. Ser. A I Math. 6, 1981, 303-342.

[V $\left.\mathrm{V}_{1}\right]$ VÄISÄL $\ddot{A}, \mathrm{~J}$.: Lectures on $n$-dimensional quasiconformal mappings. - Lecture Notes in Mathematics 229, Springer-Verlag, Berlin-Heidelberg-New York, 1971. 
[V $\left.\mathrm{V}_{2}\right]$ VÄISÄLÄ, J.: Piecewise linear approximation of lipeomorphisms. - Ann. Acad. Sci. Fenn. Ser. A I Math. 3, 1977, 377-383.

[V $\left.{ }_{3}\right]$ VÄISÄLÄ, J.: Quasi-symmetric embeddings in Euclidean spaces. - Trans. Amer. Math. Soc. 264, 1981, 191-204.

[Ve] Venema, G. A.: Approximating codimension two embeddings of cells. - Abstracts Amer. Math. Soc. 2, 1981, 590.

\author{
University of Helsinki \\ Department of Mathematics \\ SF-00100 Helsinki 10 \\ Finland
}

Received 18 August 1982 